\title{
$\alpha$-Synuclein Aggregates Increase the Conductance of Substantia Nigra Dopamine Neurons, an Effect Partly Reversed by the KATP Channel Inhibitor Glibenclamide
}

\author{
E. Hill, ${ }^{1}$ R. Gowers, ${ }^{2,3}$ M. J. E. Richardson, ${ }^{2}$ and M. J. Wall ${ }^{1}$
}

https://doi.org/10.1523/ENEURO.0330-20.2020

${ }^{1}$ School of Life Sciences, University of Warwick, Coventry CV4 7AL, United Kingdoms, ${ }^{2}$ Warwick Mathematics Institute, University of Warwick, Coventry CV4 7AL, United Kingdom, and ${ }^{3}$ Institute for Theoretical Biology, Department of Biology, Humboldt-Universität zu Berlin, Berlin 10115, Germany

\section{Visual Abstract}

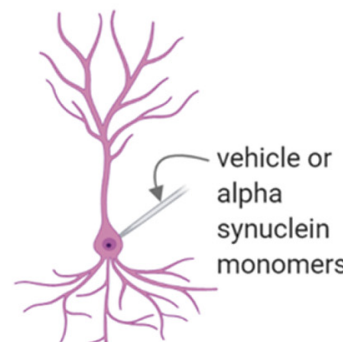

Glibenclamide-sensitive channel closed

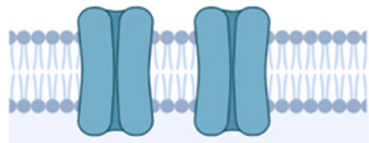

Basal Conductance

Basal Excitability

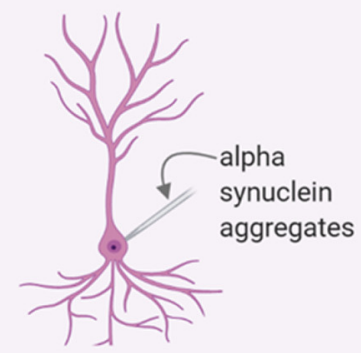

Glibenclamide-sensitive channel open

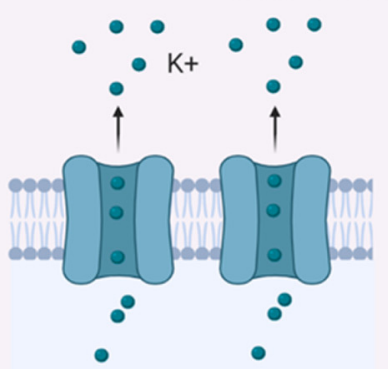

个 Conductance

$\downarrow$ Excitability

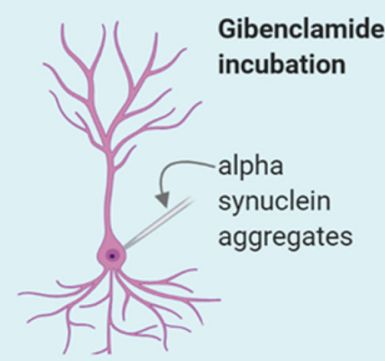

Glibenclamide-sensitive channel blocked

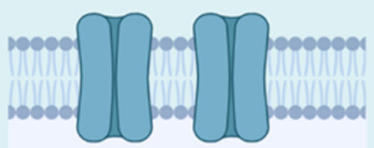

Reduced conductance change

Reduced excitability change

Dopaminergic neurons (DNs) in the substantia nigra pars compacta (SNpc) form an important part of the basal ganglia circuitry, playing key roles in movement initiation and coordination. A hallmark of Parkinson's disease (PD) is the degeneration of these SNpc DNs leading to akinesia, bradykinesia and tremor. There is gathering evidence that oligomeric $\alpha$-synuclein ( $\alpha$-syn) is one of the major pathologic species in PD, with its deposition in Lewy bodies (LBs) closely correlated with disease progression. However, the precise mechanisms 


\section{Significance Statement}

$\alpha$-Synuclein ( $\alpha$-syn) oligomers are one of the key toxic species in Parkinson's disease (PD), with their accumulation leading to dopamine neuron dysfunction. Introducing $\alpha$-syn aggregates (oligomers and possibly small fibrils) into single substantia nigra dopamine neurons led to a marked increase in whole-cell conductance and a corresponding fall in the firing rate. These changes were diminished by inhibiting ATP-sensitive $\mathrm{K}^{+}$channels (KATPs). Thus, the build-up of $\alpha$-syn oligomers during the progression of PD could chronically shunt dopamine neurons, via channel activation (which may include KATP channel activation) to reduce dopamine release.

underlying the effects of oligomeric $\alpha$-syn on DN function have yet to be fully defined. Here, we have combined electrophysiological recording and detailed analysis to characterize the time-dependent effects of $\alpha$-syn aggregates (consisting of oligomers and possibly small fibrils) on the properties of SNpc DNs. The introduction of $\alpha$-syn aggregates into single DNs via the patch electrode significantly reduced both the input resistance and the firing rate without changing the membrane potential. These effects occurred after 8-16 min of dialysis but did not occur with the monomeric form of $\alpha$-syn. The effects of $\alpha$-syn aggregates could be significantly reduced by preincubation with the ATP-sensitive $\mathrm{K}^{+}$channel (KATP) inhibitor glibenclamide. These data suggest that accumulation of $\alpha$-syn aggregates in DNs may chronically activate KATP channels leading to a significant loss of excitability and dopamine release.

Key words: $\alpha$-synuclein; dopamine; electrophysiology; KATP; patch clamp; substantia nigra

\section{Introduction}

Mid-brain dopaminergic neurons (DNs) play major roles in the control of movement, emotion, arousal and reward behavior. They possess large numbers of projections, have pacemaker activity (firing action potentials at rest) and are therefore highly energy intensive. This makes them particularly sensitive to oxidative damage (which can lead to mitochondrial dysfunction, Michel et al., 2016), and they are the most susceptible to degeneration in Parkinson's disease (PD; Damier et al., 1999). DNs are lost over the progression of PD, primarily from the substantia nigra pars compacta (SNpc) but later in the disease there is also loss from the ventral tegmental area (VTA). Symptoms of PD only become apparent when 70$80 \%$ of the dopamine input to the striatum is lost (Bernheimer et al., 1973) making it difficult to detect PD at early stages. Understanding what happens to these DNs early in pathology is important as it could provide improved diagnosis and potentially new targets to prevent or slow disease progression.

Received July 27, 2020; accepted October 18, 2020; First published November 23, 2020.

The authors declare no competing financial interests.

Author contributions: E.H., M.J.E.R., and M.J.W. designed research; E.H. performed research; E.H., R.G., and M.J.E.R. analyzed data; E.H., R.G., M.J.E.R., and M.J.W. wrote the paper.

This work was supported by Biotechnology and Biological Science Research Council-funded PhD Studentship (E.H.) an Engineering and Physical Sciences Research Council-funded PhD Studentship (R.G.).

Correspondence should be addressed to M. J. Wall at mark.wall@ warwick.ac.uk.

https://doi.org/10.1523/ENEURO.0330-20.2020

Copyright @ 2021 Hill et al.

This is an open-access article distributed under the terms of the Creative Commons Attribution 4.0 International license, which permits unrestricted use, distribution and reproduction in any medium provided that the original work is properly attributed.
$\alpha$-Synuclein ( $\alpha$-syn) is a small, native intracellular protein, found primarily at presynaptic terminals (Iwai et al., 1995) where it contributes to neurotransmitter uptake and vesicle recycling. It can also localize to the mitochondria and the nucleus (Burré et al., 2010). Molecules of $\alpha$-syn are intrinsically disordered (Alderson and Markley, 2013) but can become ordered on aggregation, triggering a pathologic cascade. First $\alpha$-syn aggregates into soluble oligomers (dimers and trimers) and then it can go on to form longer fibrils and ultimately it can form insoluble Lewy bodies (LBs; Spillantini et al., 1997). LBs are a key marker of pathology in the brains of PD patients with their abundance correlating with disease severity. LB formation involves a complex interplay between $\alpha$-syn fibrillization, posttranslational modifications, and interactions of $\alpha$-syn aggregates with membranous organelles. LB formation can lead to the disruption of mitochondrial function, to synapse dysfunction and of a decline in general cell function (Mahul-Mellier et al., 2020). However, there is also evidence that the small soluble oligomers contribute to toxicity (Winner et al., 2011; for review, see BengoaVergniory et al., 2017). At the presynaptic terminal, oligomeric $\alpha$-syn can induce synaptic dysfunction by disrupting vesicle trafficking (Jakes et al., 1994). It also interferes with synaptobrevin in the SNARE complex which is vital for the fusion of vesicles with the plasma membrane for neurotransmitter release (Murphy et al., 2000; Burré et al., 2010; Lashuel et al., 2013). Kaufmann et al. (2016) showed that $\alpha$-syn oligomers reduced the excitability and input resistance of cortical pyramidal cells.

In this study, we have used whole-cell patch-clamp recording to provide a detailed characterization of the electrophysiological effects of introducing aggregated $\alpha$-syn (oligomers plus a small proportion of fibrils) into single DNs in the substantia nigra of mouse brain slices. Since the $\alpha$-syn aggregates are only introduced into one neuron, 
there will be no compensation within the circuit and any slow cellular uptake steps (which may occur if $\alpha$-syn aggregates were applied extracellularly) are removed. Each cell acts as its own internal control, as at the time of whole-cell breakthrough the $\alpha$-syn aggregates will not have diffused into the neuron. This approach has successfully been used to examine the effects of oligomeric tau on pyramidal neurons (Hill et al., 2019) and the effects of $\alpha$-syn oligomers on Layer $\mathrm{V}$ pyramidal cells in the neocortex (Kaufmann et al., 2016).

As well as using standard current-voltage (SIV) relationships (with step-current injections) to measure the changes in DN electrophysiology, we have also utilized the dynamic IV (DIV) protocol. This was originally applied with cortical pyramidal neurons and interneurons (Badel et al., 2008a,b; Harrison et al., 2015) and can be used to develop simplified, but empirically verified quantitative models of their responses. Although the cortical cells in the original study exhibit nonlinear voltage and calciumactivated currents, under ongoing in vivo-like fluctuating current stimulation, their response could be well captured by an effective linear IV curve (away from action potential threshold). DNs, however, have more strongly expressed non-linearities (Richards et al., 1997; Neuhoff et al., 2002), and it can therefore be anticipated that the resulting DIV curves will diverge from an Ohmic linear form below threshold. Despite this, we have determined that the DIV can still be used to accurately extract a number of key electrophysiological parameters.

Using these approaches, we have characterized the real-time effects of $\alpha$-syn aggregates on the electrophysiological properties of single neurons. We find that their major effect is to induce an increase in whole-cell conductance, which significantly dampens neuronal excitability and firing rate. These effects were partially but significantly reduced by glibenclamide, an ATP-sensitive $\mathrm{K}^{+}$channel (KATP) inhibitor, suggesting a role for these channels in the pathologic actions of $\alpha$-syn aggregates.

\section{Materials and Methods}

\section{Preparation of acute brain slices}

All experiments were approved by the local Animals Welfare and Ethics Board (AWERB) at the University of Warwick. C57/BI6 mice (two to three weeks; of either sex) were killed by cervical dislocation and decapitated in accordance with the United Kingdom Animals (Scientific Procedures) Act (1986). The brain was rapidly dissected and kept on ice. The cerebellum was removed, and the rostral section of the brain was trimmed. The brain was then mounted rostral side down. Coronal slices $(350 \mu \mathrm{M})$ were cut with a Microm $\mathrm{HM} 650 \mathrm{~V}$ microslicer in cold $\left(2-4^{\circ} \mathrm{C}\right)$ high $\mathrm{Mg}^{2+}$, low $\mathrm{Ca}^{2+}$ aCSF, composed of the following: $127 \mathrm{mM} \mathrm{NaCl}$,

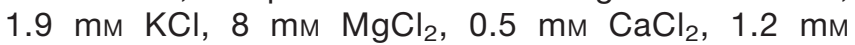
$\mathrm{KH}_{2} \mathrm{PO}_{4}, 26 \mathrm{~mm} \mathrm{NaHCO}$, and $10 \mathrm{~mm}$ D-glucose $(\mathrm{pH}$ 7.4 when bubbled with $95 \% \mathrm{O}_{2}$ and $5 \% \mathrm{CO}_{2}, 300$ mOsm). Slices were stored at $34^{\circ} \mathrm{C}$ in standard aCSF $\left(1 \mathrm{~mm} \mathrm{Mg}^{2+}\right.$ and $2 \mathrm{mM} \mathrm{Ca}^{2+}$ ) for at least $1 \mathrm{~h}$ before recording and were viable for up to $8 \mathrm{~h}$.

\section{Preparation of $\alpha$-syn aggregates}

Recombinant human $\alpha$-syn protein aggregates were purchased from Abcam (ab218819). These aggregates are advertised as preformed fibrils (PFFs) which was confirmed using negative-stain electron microscopy. Since we wanted to introduce smaller oligomeric species of $\alpha$-syn into neurons, the PFFs were first broken down into smaller aggregates before recordings were made. PFF samples were sonicated (as in Polinski et al., 2018) for $15 \mathrm{~min}(50-60 \mathrm{~Hz})$ at room temperature using a Grant Ultrasonic XUBA1 bath. Recombinant human $\alpha$-syn protein in monomeric form (ab218818) was used as a control and negative-stain electron microscopy was used to confirm it was not aggregated.

\section{Transmission electron microscopy (TEM)}

Formvar/carbon-coated 300-mesh copper grids (\#S162, Agar Scientific) were glow-discharged using the ELMO system from Cordouan Technologies. Five microliters of $\alpha$-syn species (monomer, PFF, or sonicated PFFs) were pipetted onto the grid and allowed to bind for $1 \mathrm{~min}$. Excess samples were removed with a strip of filter paper, and $5 \mu \mathrm{l}$ of $2 \%$ uranyl acetate was added for $1 \mathrm{~min}$. After removing the excess stain with a strip of filter paper, the grids were imaged using a JEOL-2100F TEM.

\section{Whole-cell patch-clamp recording}

A slice was transferred to the recording chamber, submerged and perfused $\left(2-3 \mathrm{ml} \mathrm{min}^{-1}\right)$ with aCSF at $30-32^{\circ}$ C. Slices were visualized using IR-DIC optics with an Olympus BX151W microscope (Scientifica) and a CCD camera (Hitachi). Whole-cell current-clamp recordings were made from DNs in the SNpc using patch pipettes (5$10 \mathrm{M} \Omega$ ) manufactured from thick-walled glass (Harvard Apparatus). Intracellular solution contained the following: $135 \mathrm{~mm}$ potassium gluconate, $7 \mathrm{~mm} \mathrm{NaCl}, 10 \mathrm{~mm}$ HEPES, $0.5 \mathrm{~mm}$ EGTA, $10 \mathrm{~mm}$ phosphocreatine, $2 \mathrm{~mm}$ MgATP, and $0.3 \mathrm{~mm}$ NaGTP (293 mOsm, pH 7.2). The intracellular solution was filtered $(0.2 \mu \mathrm{m})$ before the addition of $\alpha$-syn aggregates $(2 \mu \mathrm{l}$ of a $69 \mu \mathrm{m}$ stock into $275-\mu \mathrm{l}$ intracellular solution to give final concentration of $500 \mathrm{~nm}$ ) as filtering reduces the aggregate concentration (Kaufmann et al., 2016; Hill et al., 2019). The molar concentrations of $\alpha$-syn species are based on the molar mass of monomeric $\alpha$-syn (14 kDa) because of the likelihood that samples will contain a range of aggregate sizes. This method has been used in similar studies (see Hill et al., 2019; Thakur et al., 2019) and will result in an overestimate of aggregate concentration. For example, if aggregates are on average tetramers, then the concentration of aggregates will be $4 \times$ lower than reported. A subset of neurons was filled with AF594 dye $(50 \mu \mathrm{M})$ via the patch pipette for immunohistochemistry. Voltage recordings were made using an Axon Multiclamp 700B amplifier (Molecular Devices) and digitized at $20 \mathrm{kHz}$. Data acquisition and analysis were performed using pClamp 10 (Molecular Devices). Recordings from neurons that had a resting membrane potential of between -55 and $-75 \mathrm{mV}$ at whole-cell breakthrough were accepted for analysis. The bridge balance was 
Table 1: Electrophysiological parameters measured for DNs at time 0 for all experimental treatments

\begin{tabular}{|c|c|c|c|c|c|c|c|c|c|c|c|c|c|c|c|}
\hline Parameter & \multicolumn{3}{|c|}{ Vehicle/control } & \multicolumn{3}{|c|}{$\begin{array}{c}\alpha \text {-Syn } \\
\text { aggregates }\end{array}$} & \multicolumn{3}{|c|}{$\begin{array}{c}\alpha \text {-Syn } \\
\text { monomers }\end{array}$} & \multicolumn{3}{|c|}{ Glib + vehicle } & \multicolumn{3}{|c|}{$\begin{array}{l}\text { Glib }+\alpha \text {-syn } \\
\text { aggregates }\end{array}$} \\
\hline $\mathrm{RMP}(\mathrm{mV})$ & -55.8 & \pm 1.2 & \pm 3.79 & -55.4 & \pm 1.23 & \pm 4.10 & -56.8 & \pm 1.30 & \pm 3.18 & -53.2 & \pm 2.14 & \pm 5.24 & -60.4 & \pm 1.82 & \pm 4.08 \\
\hline Firing rate $(\mathrm{Hz})$ & 1.63 & \pm 0.39 & \pm 0.12 & 2.51 & \pm 1.57 & \pm 0.47 & 2.38 & \pm 1.14 & \pm 0.46 & 2.27 & \pm 1.15 & \pm 0.47 & 2.04 & \pm 1.29 & \pm 0.52 \\
\hline$C(p F)$ & 88.8 & \pm 5.74 & \pm 14.1 & 107.8 & \pm 5.84 & \pm 14.3 & 80.73 & \pm 15.1 & \pm 37.0 & 81.16 & \pm 8.54 & \pm 20.9 & 117 & \pm 22.3 & \pm 54.7 \\
\hline$g(n S)$ & 2.29 & \pm 0.2 & \pm 0.49 & 2.78 & \pm 0.2 & \pm 0.49 & 1.99 & \pm 0.3 & \pm 0.73 & 2.39 & \pm 0.29 & \pm 0.71 & 3.67 & \pm 0.55 & \pm 1.35 \\
\hline
\end{tabular}

monitored throughout the experiments and any recordings where it changed by $>20 \%$ were discarded.

\section{Stimulation protocols \\ SIV protocol}

SIVs were constructed by injecting step currents (3 s duration, with a $5 \mathrm{~s}$ interval) starting at $-200 \mathrm{pA}$ and then incrementing by either 50 or $100 \mathrm{pA}$ until a regular firing pattern was induced.

\section{Naturalistic current injection}

A naturalistic, fluctuating current (40 s in duration; for details, see below) was injected into neurons, and the resulting voltage trace was used to measure the frequency of action-potential firing (using threshold detection in Clampfit).

\section{Immunohistochemistry}

After completing the electrophysiology recordings from DNs, with the addition of AF594 dye $(50 \mu \mathrm{M})$ in the patch pipette, slices $(350 \mu \mathrm{M})$ were fixed in $4 \%$ PFA for $45 \mathrm{~min}$ at room temperature and then overnight at $4^{\circ} \mathrm{C}$. The tissue was then washed five times for $5 \mathrm{~min}$ with PBS. The slices were then blocked for $1 \mathrm{~h}$ (1\% BSA, 0.4\% Triton X-100 in PBS, $400 \mu \mathrm{l}$ per slice), then washed three times for $5 \mathrm{~min}$ with PBS. The primary antibodies against tyrosine hydroxylase (1:1000, sheep), was added to the slices $(250 \mu \mathrm{l}$ per slice) for $1 \mathrm{~h}$ at room temperature and then kept at $4-8^{\circ} \mathrm{C}$ overnight. Slices were washed five times for $5 \mathrm{~min}$ with PBS and then the secondary antibody was added (antisheep 488, 1:500, $200 \mu \mathrm{l}$ per slice) for $4 \mathrm{~h}$ at room temperature. The slices were then washed five times for $5 \mathrm{~min}$ with PBS, and then mounted on glass slides with Vectashield (Vector Laboratories). All imaging was carried using confocal microscopy (Leica 710 and Zen Black for image acquisition and processing). Controls were conducted without incubating with the primary antibodies and showed no fluorescence.

\section{Drugs and substances}

Recombinant human $\alpha$-syn aggregates were purchased from Abcam (ab218819) along with the corresponding monomers (ab218818). Dopamine (HB1835) and ZD 7288 (HB1152) were purchased from Hello Bio (HB1835). Glibenclamide (PHR1287) was purchased from SigmaAldrich. The sheep polyclonal anti-tyrosine hydroxylase was purchased from Merck (AB1542) and donkey antisheep 488 secondary from Invitrogen (A11015). The Alexa
Fluor 594 hydrazide dye was purchased from Invitrogen (10072752).

\section{Statistics}

Each recorded cell is one data point. All experimental conditions were measured using multiple animals as only one cell was recorded per slice and recording conditions were interleaved to remove bias introduced from individual animals. Data points for each experimental condition were derived from a minimum of four individual animals (the exact numbers are provided in the figure legends). Statistical analysis was performed using non parametric methods: Kruskal-Wallis one-way ANOVA, MannWhitney, and Wilcoxon signed-rank tests as required (full details provided in Table 2). All data are represented as mean and SEM with individual experiments represented by single data points. SDs are given in Table 1.

\section{Neuronal parameter extraction}

Both the SIV curve (response to constant current inputs) and DIV curve (Badel et al., 2008a,b) methods were used to extract estimates for the cellular conductance under the various pharmacological conditions. The voltage was modelled by the following the current-balance equation:

$$
C \frac{d V}{d t}+l_{\text {ion }}=l_{\text {inj }}
$$

where $C$ is the capacitance, $I_{\text {ion }}$ is the summed intrinsic current (including voltage-gated and calcium-gated currents), and $l_{i n j}$ is the current injected via the electrode during the different protocols. All computational modeling and data-fitting was conducted using custom written code in the Julia language (Bezanson et al., 2017) ported from published MATLAB code (Harrison et al., 2015).

\section{SIV curve}

To calculate neuronal resistance, the maximum voltage deflection to the $-100-p A$ current step (before the sag, Fig. $2 A$, arrow) was measured (peak neuronal resistance). The position for the voltage measurement was determined on the voltage traces at time 0 (where there was a clear peak), and then the same position was used for the rest of the voltage traces at subsequent time points.

\section{DIV curve}

As well as measuring the static properties of the neuron at rest it is also useful to measure the effective parameters when the neuronal voltage is subject to a fluctuating drive, as is the case in vivo. To this end, we employed the DIV 
Table 2: A table of all of the statistical tests and associated result

\begin{tabular}{|c|c|c|c|c|}
\hline Data & Figure & Statistical test & Test values & $p$ value \\
\hline 0 min, all conditions, RMP & 4 & Kruskal-Wallis ANOVA & $H_{(4)}=0.5385$ & $p=0.4657$ \\
\hline $0 \mathrm{~min}$, all conditions, IR & 4 & Kruskal-Wallis ANOVA & $H_{(4)}=2.109$ & $p=0.2727$ \\
\hline 0 min all conditions, FR & 4 & Kruskal-Wallis ANOVA & $H_{(4)}=1.395$ & $p=0.8511$ \\
\hline 0 vs $32 \mathrm{~min}, \alpha$-syn aggregates, IR & 4 & Wilcoxon signed-rank test & $\mathrm{W}(+)=2.0, \mathrm{~W}(-)=64.0, Z=2.7$ & $p=0.0029$ \\
\hline 0 vs 32 min, $\alpha$-syn monomers, IR & 4 & Wilcoxon signed-rank test & $W(+)=6.5, W(-)=14.5, Z=0.73$ & $p=0.4688$ \\
\hline 0 vs 32 min, vehicle, IR & 4 & Wilcoxon signed-rank test & $W(+)=46.0, W(-)=9.0, Z=-1.83$ & $p=0.0645$ \\
\hline $32 \mathrm{~min}, \alpha$-syn aggregates, $\alpha$-syn monomers and vehicle, IR & 4 & Kruskal-Wallis ANOVA & $H_{(2)}=14.53$ & $p=0.0007$ \\
\hline $16 \mathrm{~min}, \alpha$-syn aggregates, $\alpha$-syn monomers and vehicle, IR & 4 & Kruskal-Wallis ANOVA & $H_{(2)}=10.67$ & $p=0.0048$ \\
\hline 0 vs $32 \mathrm{~min}$, vehicle, RMP & 4 & Wilcoxon signed-rank test & $W(+)=41.5, W(-)=3.5, Z=-2.19$ & $p=0.0234$ \\
\hline 0 vs 32 min, $\alpha$-syn aggregates, RMP & 4 & Wilcoxon signed-rank test & $W(+)=61.0, W(-)=5.0, Z=-2.44$ & $p=0.0107$ \\
\hline 0 vs 32 min, $\alpha$-syn monomers, RMP & 4 & Wilcoxon signed-rank test & $W(+)=19.5, W(-)=1.5 Z=-1.79$ & $p=0.0938$ \\
\hline $32 \mathrm{~min}, \alpha$-syn aggregates, $\alpha$-syn monomers and vehicle, RMP & 4 & Kruskal-Wallis ANOVA & $H_{(2)}=2.234$ & $p=0.3273$ \\
\hline 0 vs $32 \mathrm{~min}$, vehicle, FR & 4 & Wilcoxon signed-rank test & $W(+)=14, W(-)=41.0 Z=-1.32$ & $p=0.1895$ \\
\hline 0 vs $32 \mathrm{~min}, \alpha$-syn aggregates, FR & 4 & Wilcoxon signed-rank test & $W(+)=1.0, W(-)=65.0 Z=2.8$ & $p=0.0020$ \\
\hline 0 vs 32 min, $\alpha$-syn monomers, FR & 4 & Wilcoxon signed-rank test & $W(+)=11, W(-)=10.0 Z=0$ & $p>0.9999$ \\
\hline $32 \mathrm{~min}, \alpha$-syn aggregates, $\alpha$-syn monomers and vehicle, FR & 4 & Kruskal-Wallis ANOVA & $H_{(2)}=10.21$ & $p=0.0061$ \\
\hline 0 min, all conditions, capacitance & 5 & Kruskal-Wallis ANOVA & $H_{(4)}=6.269$ & $p=0.18$ \\
\hline 0 vs $32 \mathrm{~min}, \alpha$-syn aggregates, membrane conductance & 5 & Wilcoxon signed-rank test & $W(+)=66.0, W(-)=0 Z=-2.89$ & $p=0.001$ \\
\hline 0 vs $32 \mathrm{~min}$, vehicle, membrane conductance & 5 & Wilcoxon signed-rank test & $W(+)=71.0, W(-)=7 Z=-2.47$ & $p=0.009$ \\
\hline $32 \mathrm{~min}, \alpha$-syn monomers and vehicle, membrane conductance & 5 & Mann-Whitney test & $\begin{array}{l}\text { Vehicle median }=2.820, n=12, \alpha \text {-syn monomers }=2.820 \text {, } \\
\qquad n=6, U=36\end{array}$ & $p>0.9999$ \\
\hline $32 \mathrm{~min}, \alpha$-syn aggregates and vehicle, membrane conductance & 5 & Mann-Whitney test & $\begin{array}{l}\alpha \text {-Syn aggregates median }=6.450, n=11 \text {, vehicle median }=2.820 \\
\quad n=12, U=11\end{array}$ & $p=0.0003$ \\
\hline 0 min, vehicle and vehicle + glib, RMP & 6 & Mann-Whitney test & $\begin{array}{l}\text { Vehicle median }=-56, n=10 \text {, vehicle }+ \text { glib median }=-54, n=6 \text {, } \\
\qquad U=21.50\end{array}$ & $p=0.3791$ \\
\hline 0 min, vehicle and vehicle + glib, IR & 6 & Mann-Whitney test & $\begin{array}{l}\text { Vehicle median }=338.3, n=10 \text {, vehicle }+ \text { glib median }=393, n=6 \text {, } \\
\qquad U=18.50\end{array}$ & $p=0.2294$ \\
\hline $0 \mathrm{~min}$, vehicle and vehicle + glib, FR & 6 & Mann-Whitney test & $\begin{array}{l}\text { Vehicle median }=69.5, n=10 \text {, vehicle }+ \text { glib median }=86.5, n=6 \text {, } \\
\qquad U=25.50\end{array}$ & $p=0.6548$ \\
\hline 0 vs 32 min, vehicle + glib, IR & 6 & Wilcoxon signed-rank test & $W(+)=9.0, W(-)=12.0 Z=0.21$ & $p=0.8438$ \\
\hline 0 vs 32 min, vehicle + glib, FR & 6 & Wilcoxon signed-rank test & $\mathrm{W}(+)=7.0, \mathrm{~W}(-)=14.0 Z=0.63$ & $p=0.5625$ \\
\hline 0 vs 32 min, $\alpha$-syn + glib, IR & 6 & Wilcoxon signed-rank test & $W(+)=5.0, W(-)=16.0 Z=1.04$ & $p=0.25$ \\
\hline 0 vs 32 min, $\alpha$-syn + glib, FR & 6 & Wilcoxon signed-rank test & $\mathrm{W}(+)=7.0, \mathrm{~W}(-)=14.0 Z=0.63$ & $p=0.353$ \\
\hline 0 vs 32 min, $\alpha$-syn + glib, membrane conductance & 7 & Wilcoxon signed-rank test & $W(+)=21.0, W(-)=0 Z=0.63$ & $p=0.0313$ \\
\hline $32 \mathrm{~min}$, vehicle + glib and $\alpha$-syn + glib, membrane conductance & 7 & Mann-Whitney test & $\begin{array}{l}\text { Vehicle }+ \text { glib median }=4.675, n=6, \alpha \text {-syn }+ \text { glib median }=3.33 \\
\qquad n=6, U=11\end{array}$ & $p=0.3095$ \\
\hline Normalized conductance over time, all conditions & 7 & Kruskal-Wallis ANOVA & $F_{(4,180)}=6.806$ & $p<0.0001$ \\
\hline Conductance effect & & Dunn's post hoc analysis & $F_{(4,180)}=11.01$ & $p<0.0001$ \\
\hline Time effect & & & & $p<0.0001$ \\
\hline$\alpha$-Syn aggregates vs control & & & & $p=0.0003$ \\
\hline$\alpha$-Syn aggregates vs monomers & & & & $p<0.0001$ \\
\hline$\alpha$-Syn aggregates vs vehicle + glib & & & & $p=0.0045$ \\
\hline$\alpha$-Syn aggregates vs $\alpha$-syn + glib & & & & \\
\hline
\end{tabular}

curve protocol (Badel et al., 2008) in which a stochastic current $l_{\text {inj }}$ is injected into the cell to induce a fluctuating voltage response and triggering of action potentials (Fig. $2 B$ ). A detailed description of the protocol has been previously published (Badel et al., 2008) as well as the computer code required (Harrison et al., 2015). In brief, a noisy current trace $l_{\text {inj }}$ is generated computationally (from the sum of two Ornstein-Uhlenbeck Gaussian processes with time constants $\tau_{\text {fast }}=3 \mathrm{~ms}$ and $\tau_{\text {slow }}=10 \mathrm{~ms}$ to mimic AMPA and GABA receptor time courses) and injected into the cell with the resultant voltage measured. The voltage Equation 1 can be rearranged for the ionic current to give:

$$
I_{\text {ion }}=I_{\text {inj }}-C \frac{d V}{d t},
$$

where the injected current is known, and the derivative can be calculated from measured voltage (with the capacitance extracted using the method in Badel et al., 2008). The ionic current and voltage time courses can then be plotted against each other (Fig. $2 C$, red scattered points). Given the interaction of the gated ionic currents with the stochastic injected current, there is significant scatter in the relation. However, the ionic current can be averaged in voltage slices to provide the DIV curve (Fig. $2 C$, black solid line, $D$, expanded view). Once the DIV curve has been obtained the various features can be extracted such as the ohmic conductance, the linear component of the IV curve at more hyperpolarized subthreshold voltage, and the excess depolarization-activated current seen in these cells near threshold.

By using both DIV and SIV we use two independent methods that induce distinct voltage dynamics to extract neuronal parameters thereby increasing the robustness of the data. These approaches also examine the neurons under different conditions. For SIV, the parameters are 
extracted from neurons that are in a simple dynamical state. In contrast the fluctuations induced by the DIV protocol result in a voltage that rapidly explores a much larger range.

\section{Results}

\section{Characterizing DNs in the substantia nigra}

Whole-cell patch-clamp recordings were made from putative DNs located in the SNpc (identified by position in the slice). Neurons were initially confirmed to be SNpc DNs from their electrophysiological profile, which was consistent with previous studies (Grace and Onn, 1989; Richards et al., 1997; Krashia et al., 2017). Most of the recorded neurons displayed slow autonomous firing (36 out of 49 neurons, $73 \%$, mean firing rate of $1.05 \pm 0.082 \mathrm{~Hz}$; Fig. 1A) consistent with the reported properties for dopamine neurons in the SNpc (Grace and Onn, 1989). These neurons were sensitive to dopamine (Lacey et al., 1989), with application of dopamine (30 $\mu \mathrm{m})$ hyperpolarizing the membrane potential by $7.35 \pm 1.24 \mathrm{mV}$ and abolishing the spontaneous firing (Fig. $1 A, n=8$ ). The neurons showed a characteristic (Neuhoff et al., 2002) response to step-current injection (Fig. 1B), with a large sag in response to hyperpolarizing current steps (Fig. 1C) indicative of the presence of $\mathrm{I}(\mathrm{h})$. Following the termination of hyperpolarizing-current steps there was a rebound potential leading to firing in 33 out of 49 neurons (68\%; Fig. 1C) another characteristic of I(h). Dopamine reduced the firing in response to the positive current steps and to the naturalistic current injection (firing rate reduced to $62.7 \pm 0.2 \%$ of that in control). Dopamine also reduced the voltage response to hyperpolarizing current steps (indicative of an increase in whole-cell conductance) and also abolished the rebound firing (Fig. 1D). Application of ZD7288 (100 $\mu \mathrm{M})$, a pharmacological inhibitor of I(h) in dopamine neurons (Harris and Constanti, 1995), greatly reduced the sag response produced by hyperpolarizing current steps and also abolished the rebound firing, confirming that they were $\mathrm{I}(\mathrm{h})$ mediated (Fig. $1 E, n=6)$. Finally, to confirm that the neurons were dopaminergic, a subset of the recorded neurons $(n=9)$ were filled with AF594 dye via the patch pipette and immunofluorescence staining was used to confirm that they were tyrosine hydroxylasepositive (Fig. 1F).

\section{Detailed analysis of the electrophysiology of DNs in the substantia nigra}

We used two approaches to characterize changes that occurred when SNpc DNs had either $\alpha$-syn aggregates, monomers, or vehicle introduced. The first constituted of measurements from SIV curve protocols during which neurons received constant-current (step) inputs (Fig. 2A). The second approach measures neuronal properties using the DIV curve protocol (Badel et al., 2008a,b; Harrison et al., 2015), during which neurons were stimulated by a stochastic current mimicking fluctuating synaptic drive (Fig. 2B-D). This methodology allows for the average ionic current at a particular voltage to be measured at a good resolution over the full voltage range. The resulting DIV curve can be used to provide capacitance, ohmic conductance at hyperpolarized regimes as well as the strength of excess current seen near threshold in these cells (Fig. 2B-D). It is clear that the DIV curve diverges from linear at potentials above $\sim-50 \mathrm{mV}$ (but below action-potential threshold) which is characteristic of a depolarization-activated outward current (Fig. 2D). This effect of the outward current can be isolated by subtracting an extrapolated fit to the linear portion of the DIV curve (Fig. 2D, inset).

\section{Characterizing the structure of the $\alpha$-syn aggregates}

Recombinant human $\alpha$-syn protein was purchased from Abcam in the form of PFF aggregates (ab218819) and in the form of monomers (ab218818). Negative stain TEM was initially to confirm that the samples were either monomeric (Fig. 3B) or PFFs (Fig. 3C). To enable delivery of aggregates, mostly in oligomeric form, via the patch pipette, the PFFs were broken down. We used sonication (as in Polinski et al., 2018) for 15 min (50-60 Hz). We compared the structure of the $\alpha$-syn aggregates (sonicated PFFs) to oligomeric tau which had an annular structure (Fig. 3A; Hill et al., 2019) to ensure that it consisted of oligomeric species (Fig. 3B). These oligomeric forms were stable for at least $3 \mathrm{~h}$ on ice. While the majority of the species in our samples had an oligomeric structure, we cannot exclude the possibility that other forms of aggregates exist (for example, small $\sim 50-n m$ fibrils; Polinski et al., 2018). We therefore use the term aggregates rather than oligomers. Monomeric or aggregated $\alpha$-syn samples were introduced into DNs via the patch pipette.

\section{$\alpha$-Syn aggregates but not monomers have marked effects on the electrophysiological properties of SN DNs}

We introduced either aggregated or monomeric $\alpha$-syn (500 $\mathrm{nM}$, same concentration as Kaufmann et al., 2016) via the patch pipette during whole-cell current-clamp recording from identified SNpc DNs. To ensure consistency, all molar concentrations were based on the molar mass of monomeric $\alpha$-syn as the samples probably contain a range of aggregate sizes (see above). Electrophysiological parameters were then measured from the voltage responses to step currents (SIV) and naturalistic current injections (DIV). The currents for SIV and DIV were injected at 8 min intervals for the duration of recordings (32 min; Kaufmann et al., 2016). In control experiments, the same volume of vehicle (2- $\mu$ PBS) was added to the intracellular solution. Recordings ( $\alpha$-syn aggregates, monomers, or vehicle) were made from SNpc DNs in interleaved slices to minimize variation.

At time 0 (a few minutes following whole-cell breakthrough), there was no significant difference in the measured parameters from SIVs (membrane potential $p=0.4657$, resistance $p=0.2747$ and firing rate $p=0.8511$; Table 1) between neurons which had received either vehicle, $\alpha$-syn monomers or aggregates. Thus, the recording quality and neuronal properties were comparable between the experimental groups. However, as illustrated in the example in Figure $4 A$, there were marked changes in the SIV during recording from 
A

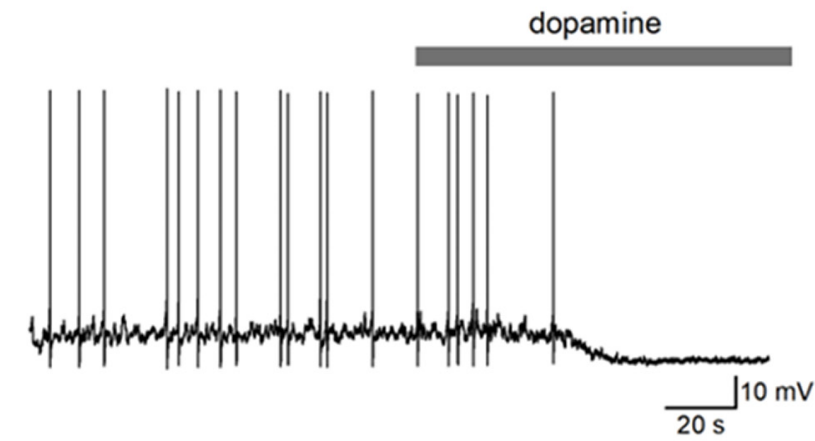

C

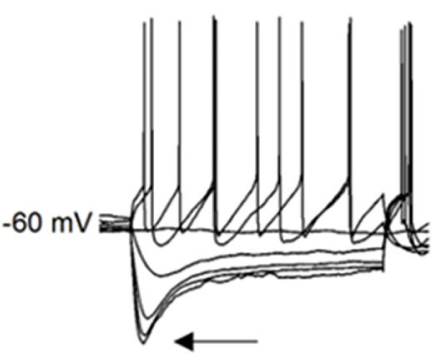

D
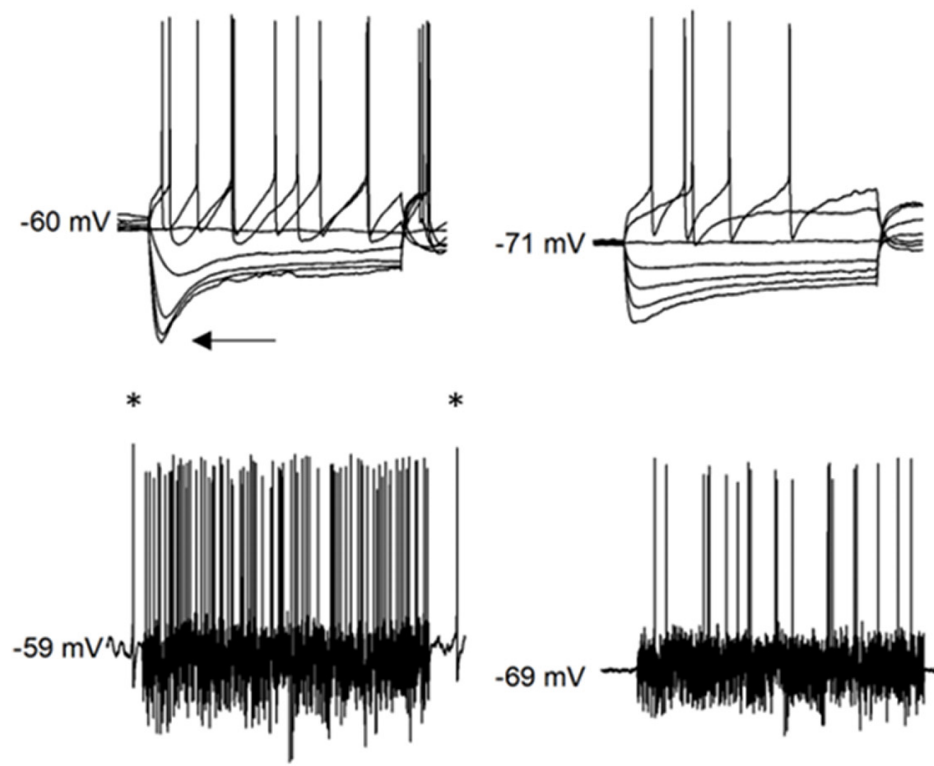

$\mathbf{F}$ AF 594 filled cell

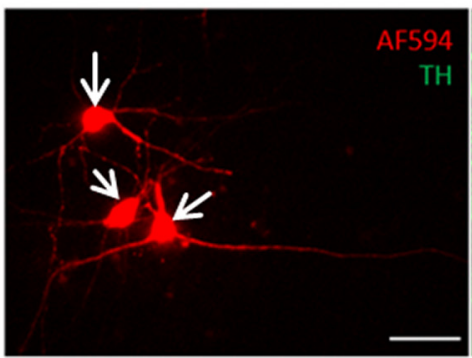

$-69 \mathrm{mV}$

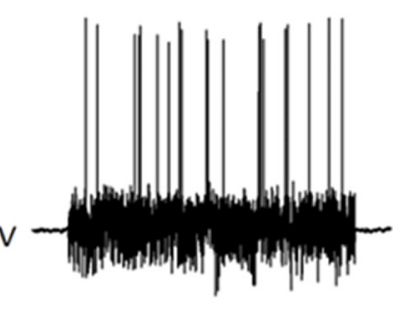

tyrosine hydroxylase

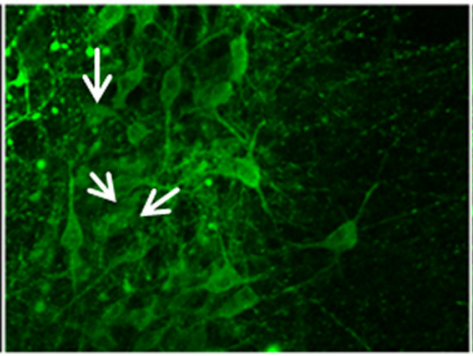

B SIV Injected current
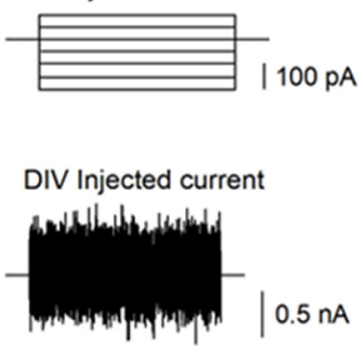

E

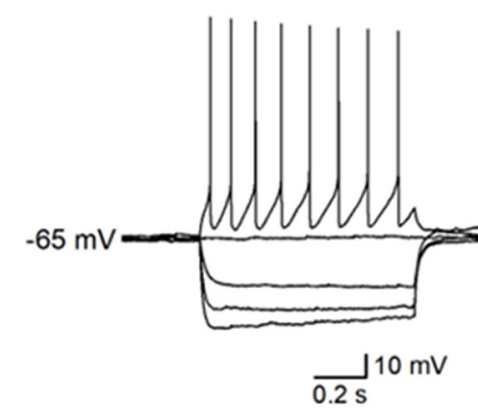

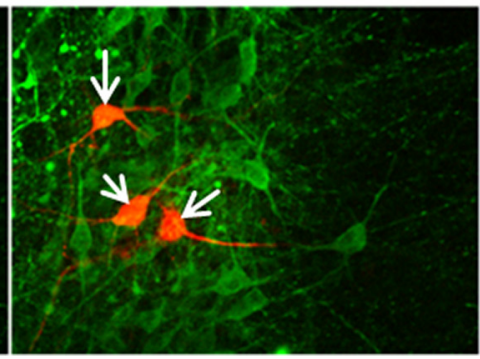

Figure 1. Whole-cell patch-clamp recording from DNs in the SNpc. $\boldsymbol{A}$, Example membrane potential trace recorded from a putative $\mathrm{DN}$ in the substantia nigra which showed characteristic spontaneous action potential firing. Dopamine (30 $\mu \mathrm{M})$ hyperpolarized the neuron from -62 to $-70 \mathrm{mV}$ and stopped the action potential firing. $\boldsymbol{B}$, The different input currents delivered to putative DNs. SIV is the standard step current protocol, $3 \mathrm{~s}$ duration steps starting at $-200 \mathrm{pA}$ and increasing by $50 \mathrm{pA}$ until the neuron exhibits a regular firing pattern. DIV uses a fluctuating naturalistic noisy current trace (for details, see Materials and Methods) which is also used to determine action potential firing rate. $\boldsymbol{C}$, top, Membrane potential traces in response to current steps. The recorded neuron displays characteristic features of DNs: a large sag in response to hyperpolarizing steps (arrow) and rebound firing ( $\left(^{*}\right)$. Bottom, Membrane potential trace from the same cell in response to naturalistic current injection. The neuron can be seen to be firing at rest $\left(^{\star}\right)$ which is a characteristic feature of these neurons. $\boldsymbol{D}$, top, Membrane potential traces in response to current steps following application of dopamine $(30 \mu \mathrm{M})$. The sag is reduced, the membrane potential hyperpolarized, the firing reduced, and the rebound firing is absent. Bottom, Membrane potential trace from the same cell in response to naturalistic current injection in dopamine (30 $\mu \mathrm{M})$. The neuron has stopped firing at rest, fires less frequently during current application and is hyperpolarized. $\boldsymbol{E}$, top, Membrane potential traces in response to current steps in the presence of the I(h) blocker ZD7288 (100 $\mu \mathrm{M})$ are similar to that previously reported for DN (Harris and Constanti, 1995). The sag response to hyperpolarizing current steps is markedly reduced, the resting membrane potential is hyperpolarized, the firing rate reduced and the voltage response following the spike is altered. Bottom, Membrane potential response 
continued

of the same neuron to the naturalistic injected current in ZD7288 (100 $\mu \mathrm{M})$. $\boldsymbol{F}$, Tyrosine hydroxylase (TH) immunohistochemistry confirms that the recorded neurons were DNs. Neurons were filled with AF594 dye (red) via the patch pipette and then slices were stained for TH (green). The merged image shows that the recorded neurons express TH and are therefore dopaminergic. Scale bar: $50 \mu \mathrm{m}$.

neurons in which $\alpha$-syn aggregates had been introduced. The changes in SIV, reduction in voltage responses and decrease in firing rate at positive potentials, were indicative of a significant fall in neuronal resistance and the opening of a membrane channel. Such large changes in the SIV did not occur in the neurons that received either $\alpha$-syn monomers or vehicle (Fig. 4B,C). For the neurons injected with $\alpha$-syn aggregates, after $32 \mathrm{~min}$ of recording, the resistance was significantly $(p=0.0029, n=11)$ reduced to $63 \pm 9.21 \%$ of the resistance measured at $0 \mathrm{~min}$ (Fig. 4D). There was no significant changes in the resistance for neurons injected with either $\alpha$-syn monomers $(p=0.4688, n=6)$ or vehicle $(p=0.0645$, $n=10$ ) over the duration of recordings (at 32 min input resistance $(\mathrm{IR})$ was $94 \pm 5 \%$ of the resistance measured at time 0 min for vehicle and $99 \pm 9 \%$ of resistance measured at time 0 min for monomers; Fig. 4D). At the 32-min time point there was a significant $(p=0.0007)$ difference in resistance between the neurons that received $\alpha$-syn monomers or vehicle to those where $\alpha$-syn aggregates were introduced. We investigated the time at which $\alpha$-syn aggregates began to affect neuronal resistance and found that the difference in resistance between control and aggregated and monomeric $\alpha$-syn neurons first became significant at the 16-min time point $(p=0.0048$; Fig. 4D). Thus, the resistance started to fall (presumably as membrane channels opened) between 8 and 16 min after $\alpha$-syn aggregate introduction into the neuron at whole-cell break through.

The recorded neurons had a membrane potential of around $-55 \mathrm{mV}$ (see Table 1) at the start of recordings. For all experimental conditions, the membrane potential slowly and weakly depolarized over the duration of the recordings (Fig. 4E). For vehicle, the mean depolarization $(\Delta \mathrm{Vm})$ was $6.2 \pm 1.82 \mathrm{mV}$ after $32 \mathrm{~min}$ of recording $(p=$ $0.0234, n=10)$, for $\alpha$-syn monomers $\Delta \mathrm{Vm}$ was $3.8 \pm 1.2$
$\mathrm{mV}(p=0.0938, n=6)$, and for $\alpha$-syn aggregates $\Delta \mathrm{Vm}$ was $6.8 \pm 1.85 \mathrm{mV}(p=0.0107, n=11)$. There was no statistically significant difference between the membrane potentials of the control (vehicle and monomers) versus $\alpha$-syn aggregates across the duration of the recordings $(p=$ $0.3273)$. Thus, although $\alpha$-syn aggregates markedly reduced neuronal resistance they did not produce a significant change in the membrane potential.

For control neurons (vehicle), although the membrane potential was depolarized, the change in firing rate did not reach significance $(p=0.1895$, measured from the naturalistic current injection, firing rate at $32 \mathrm{~min}$ was $124.7 \pm 1.3 \%$ of the firing rate at time $0 \mathrm{~min}, n=10$; Fig. $4 F, G)$. For neurons which received $\alpha$-syn monomers, although the membrane potential was depolarized there was also no significant $(p>0.9999)$ change in the firing rate (measured from the naturalistic current injection, firing rate at $32 \mathrm{~min}$ was $120.4 \pm 2.4 \%$ of the firing rate at time $0 \mathrm{~min}, n=6$; Fig. $4 F, G$ ). For the neurons which had $\alpha$-syn aggregates introduced, there was a significant $(p=0.0020)$ reduction in the firing rate (at 32 min the firing rate was $42.3 \pm 9.3 \%$ of the firing rate measured at $0 \mathrm{~min}$, $n=11$; Fig. 4F,G). When comparing control (vehicle and monomers) versus $\alpha$-syn aggregates there was a significant difference in the firing rate at $32 \min (p=0.0061)$. This fall in firing rate induced by $\alpha$-syn aggregates is consistent with the marked fall in neuronal resistance.

$\alpha$-Syn aggregates decreased the occurrence of rebound firing following the termination of hyperpolarization steps $[73 \%$ of neurons $(8 / 11)$ showed rebound firing at 0 min, but only $18 \%(2 / 11)$ till showed it after 32 min of recording]. In contrast, all the control neurons that initially displayed rebound firing [60\% (6/10)] still showed it after 32 min of recording. Aggregated $\alpha$-syn also reduced the
A

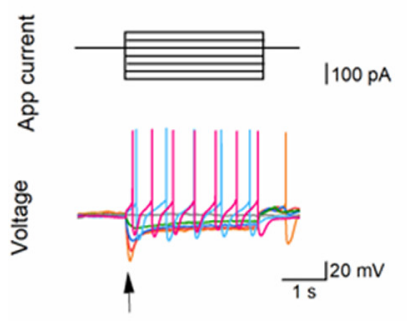

B

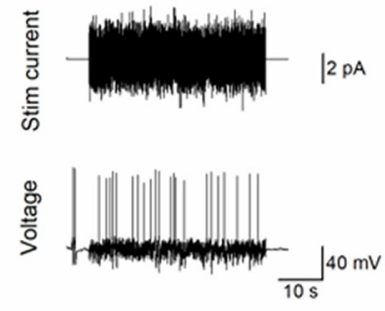

C

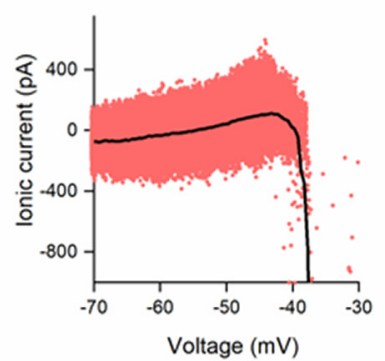

D

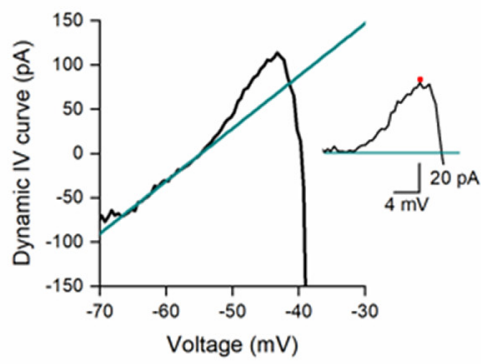

Figure 2. Extracting neuronal parameters using SIV $(\boldsymbol{A})$ and DIV $(\boldsymbol{B}-\boldsymbol{D})$ methodologies. For the SIV measurements: $(\boldsymbol{A})$ applied current and voltage response. Arrow shows location of voltage measurement for the calculation of neuronal resistance (peak voltage deflection). For the DIV measurements: $(\boldsymbol{B})$ naturalistic stimulation current and voltage response. $\boldsymbol{C}$, The estimated ionic current as a scatter plot against voltage (points). The DIV curve (black) is the typical ionic current at a particular voltage where data within $200 \mathrm{~ms}$ postspike was not included to avoid the effects of transitory spike-generated currents. $\boldsymbol{D}$, The DIV curve (black) and its linear fit to the ohmic component (green, from hyperpolarized voltages to the resting voltage). Inset, Isolated outward current. For further details of the SIV and DIV fitting procedures, see Materials and Methods. 


\section{A tau oligomers}

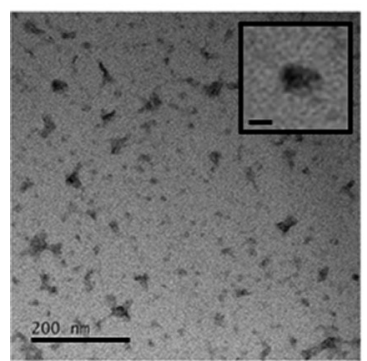

B aSyn PFFs

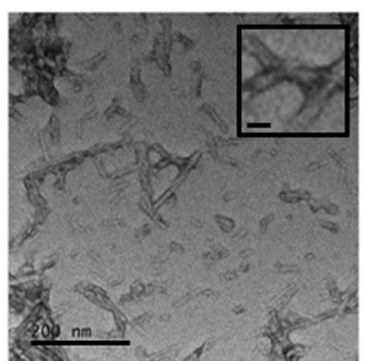

C aSyn oligomers

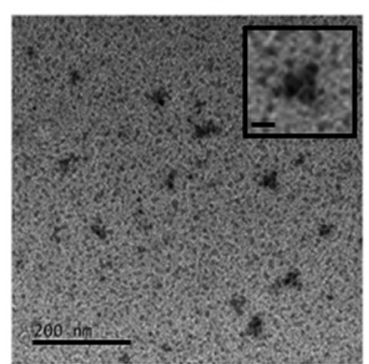

D aSyn monomers

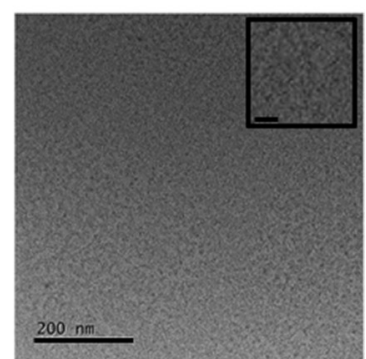

Figure 3. Structural analysis of $\alpha$-syn samples. Electron micrographs of negative-stain-TEM transmission electron microscopy analyzed protein samples. The sample $(5 \mu \mathrm{l})$ was applied onto a copper grid and fixed with uranyl acetate. Magnification is $60,000 \times$. $\boldsymbol{A}$, Tau aggregates (for reference) showing aggregated form. Inset, Higher magnification showing annular form of aggregates. $\boldsymbol{B}, \alpha$-Syn monomers show no aggregation. Inset, Higher magnification showing no structural form. $\boldsymbol{C}, \alpha$-Syn aggregates display many large fibrils (PFFs). Inset, Example of fibrils at higher magnification. $\boldsymbol{D}, \alpha$-Syn fibrils were added to intracellular patch solution and then sonicated for $15 \mathrm{~min}$ to form small aggregates. Inset, Annular form of aggregates. Scale bar: $200 \mathrm{~nm}, 20 \mathrm{~nm}$.

occurrence of tonic firing [64\% of neurons $(7 / 11)$ were initially spontaneously active but after 32 min only $27 \%(3 /$ 11 ) of neurons were still active]. For control neurons, $80 \%$ $(8 / 10)$ initially displayed tonic firing, with $60 \%(6 / 10)$ still active after $32 \mathrm{~min}$ of recording. We examined at what time the neurons stopped spontaneously firing and found that on average control (vehicle) neurons that ceased firing stopped firing at $26 \pm 3.7 \mathrm{~min}$, which was similar to the time for neurons that received $\alpha$-syn monomers (28 \pm $3.64 \mathrm{~min})$. The neurons that received $\alpha$-syn aggregates stopped firing significantly earlier $(15.1 \pm 2.33 \mathrm{~min})$ than either control (vehicle) neurons $(p=0.0274)$ or neurons that received $\alpha$-syn monomers $(p=0.0392)$.

\section{Further analysis of the effects of $\alpha$-syn aggregates on DNs using the DIV curve}

In control neurons (vehicle) the DIV curve did not markedly change throughout the duration of recordings (Fig. $5 A)$. In contrast $\alpha$-syn aggregates induced significant changes to the DIV, in particular increasing the slope (Fig. $5 B$ ). These effects were not observed with $\alpha$-syn monomers (Fig. 5C). For all experimental conditions, there were no significant changes in the cellular capacitance $(p=0.18$; Fig. $5 E)$. Thus, the introduction of $\alpha$-syn aggregates had no effect on the electrotonic properties of the neurons, by, for example, electrically isolating compartments. Aggregated $\alpha$-syn markedly increased the membrane conductance $(272 \%$ of the conductance at $0 \mathrm{~min}$ by $32 \mathrm{~min}, p=0.001$; Fig. $5 D$ ). In control neurons (vehicle or $\alpha$-syn monomers), there was a small increase in conductance (vehicle conductance was $135 \%$ of the conductance at 0 min by $32 \min p=0.009 ; \alpha$-syn monomer conductance was $141.2 \%$ of the conductance at 0 min by $32 \mathrm{~min}, \mathrm{~ns})$ over the duration of recordings. At $32 \mathrm{~min}$ there was no significant $(p>0.9999)$ difference between the conductance of vehicle and $\alpha$-syn monomer treated neurons. In contrast, the conductance of $\alpha$-syn aggregate treated neurons was significantly $(p=0.0003)$ larger than vehicle treated neurons. There were no significant differences in the other extracted parameters: resting membrane potential, spike potential or spike-resting potential between the three experimental conditions (Fig. 5F-H). Thus, the reduction in excitability induced by $\alpha$-syn aggregates is only because of a change in conductance rather than changes in, for example, the potential difference between rest and threshold.

\section{The decrease in conductance and firing rate caused by $\alpha$-syn is in part because of the opening of KATP channels}

Previous studies have shown that either blocking KATP or genetically deleting them has protective effects on DNs in PD rodent models (Liss et al., 2005; Zhang et al., 2018). There has also been a recent preprint that shows that the effects of $\alpha$-syn aggregates on spontaneous firing can be prevented by blocking KATP channels (Thakur et al., 2019). To investigate whether the increase in membrane conductance that we have observed could be prevented by inhibiting KATP channel opening, we used the classic KATP blocker glibenclamide (Light and French, 1994; Jiang and Haddad, 1997). We first investigated whether the presence of glibenclamide throughout recordings had any effect on the electrophysiological properties of SNpc DNs (Fig. 6A). There was no significant difference in any of the measured parameters (from SIVs, membrane potential $p=0.3791$, resistance $p=0.2294$, firing rate $p=0.6548$ ) at 0 min between neurons from slices which had been preincubated with glibenclamide $(1 \mu \mathrm{M}, n=6)$ compared with neurons in control slices (both with vehicle in the patch pipette). These neuronal parameters did not significantly change over the duration of the recordings with glibenclamide present: resistance (after $32 \mathrm{~min} 99 \pm 7.5 \%$ of the resistance at time $0 \mathrm{~min}$, $p=0.08,438, n=6$ ) or firing rate (after $32 \min 112.1 \pm 26.6 \%$ of the firing rate at time 0 min, $p=0.5625$; Fig. $6 A$ ). Thus, glibenclamide had no significant effect on neuronal properties measured from SIVs that could occlude the actions of $\alpha$-syn aggregates.

We then made whole-cell recordings with $\alpha$-syn aggregates in the patch pipette in slices preincubated in glibenclamide (1 $\mu \mathrm{m}$; Fig. $6 B)$. The fall in resistance and firing rate that was observed in the presence of $\alpha$-syn aggregates did not occur when the slices were incubated with 
A

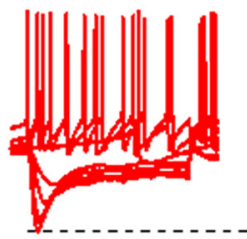

B
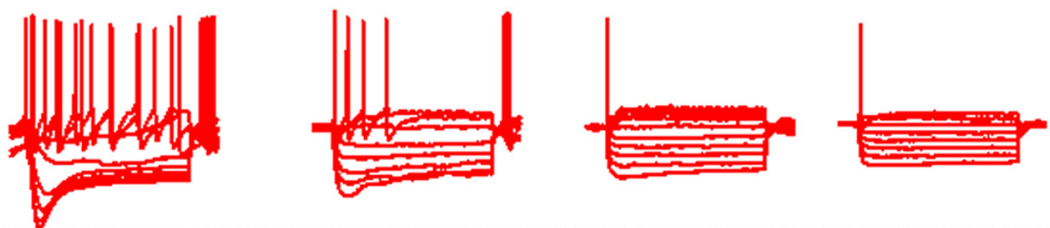

$-20 \mathrm{mV}$
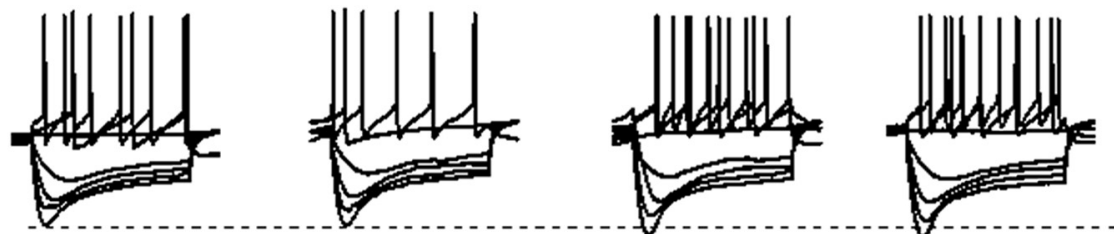

$0.2 \mathrm{~s}$

C

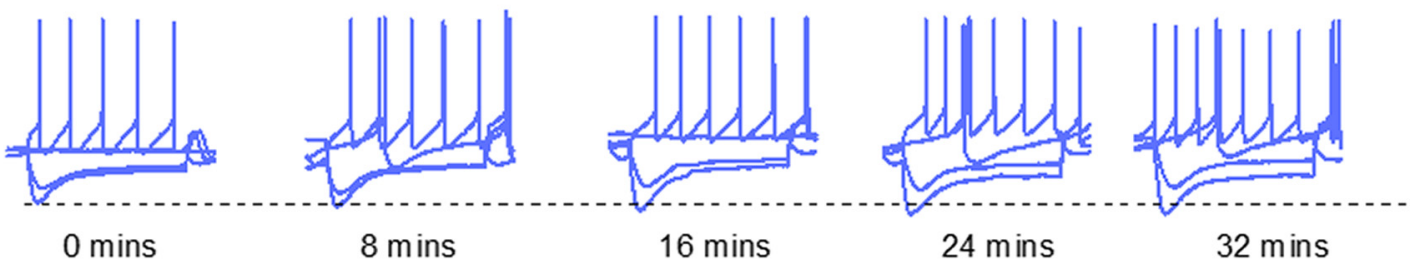

D

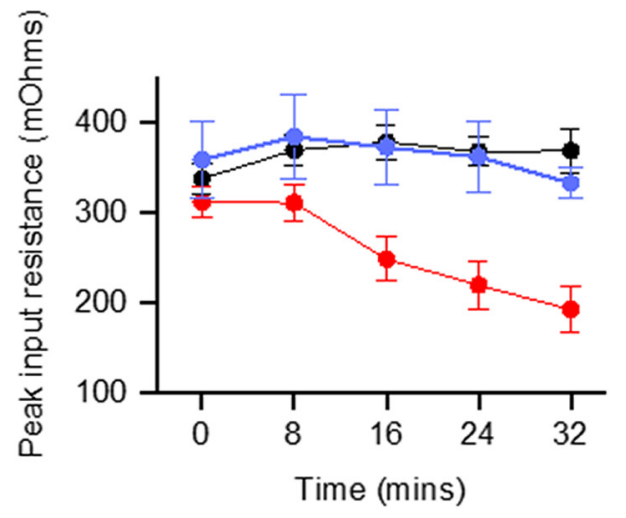

$\mathbf{F}$

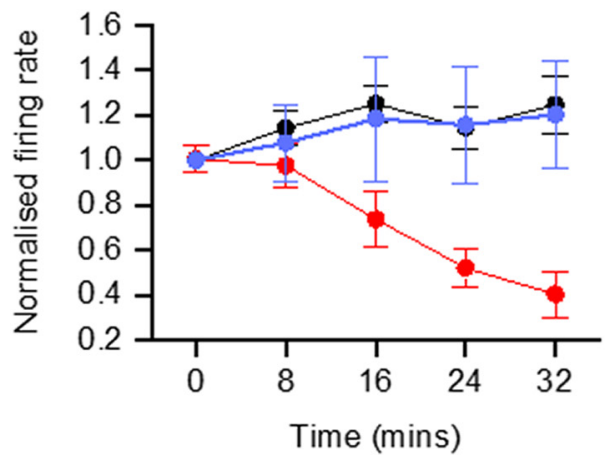

E

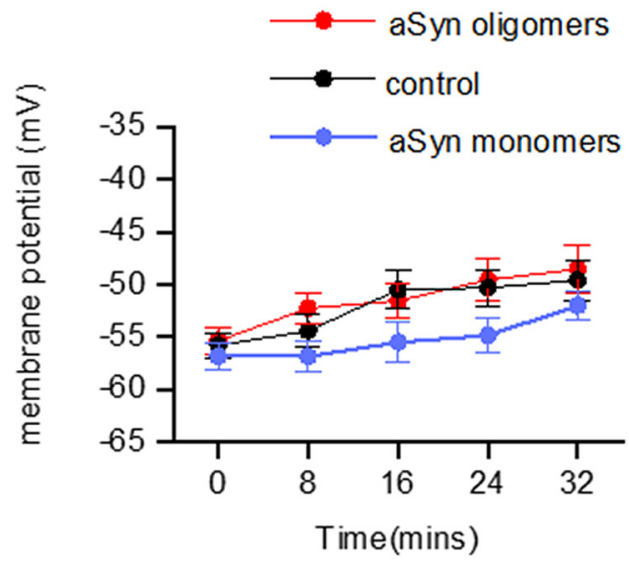

G

0 mins
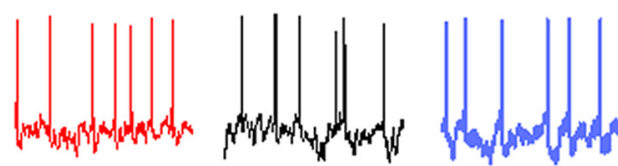

32 mins
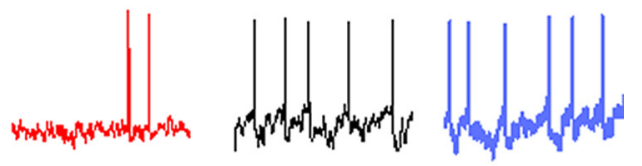

$\overrightarrow{0.5 \mathrm{~s}}$

Figure 4. $\alpha$-Syn aggregates induce a time-dependent decline in firing rate and significant fall in neuronal resistance. Example current-voltage relationships (SIV) for DNs injected with $\alpha$-syn aggregates $(\boldsymbol{A})$, vehicle $(\boldsymbol{B})$, and $\alpha$-syn monomers $(\boldsymbol{C})$. Current steps (starting at $-200 \mathrm{pA}$ and increasing by 50 or $100 \mathrm{pA}$ until a regular firing pattern was induced) were used to construct SIVs. Recordings display SIVs at time points between whole-cell breakthrough ( $0 \mathrm{~min})$ and up to $32 \mathrm{~min}$. The neuron which had the $\alpha$-syn aggregates introduced $(\boldsymbol{A})$ shows a clear reduction in the voltage responses and a fall in the firing rate at positive potentials, whereas the neurons that received either vehicle or $\alpha$-syn monomers show no significant changes $(\boldsymbol{B}, \boldsymbol{C})$. $\boldsymbol{D}$, Mean neuronal resistance plotted against time for control (vehicle) neurons and neurons that had either $\alpha$-syn aggregates or monomers introduced. For the 
continued

neurons injected with $\alpha$-syn aggregates, after $32 \mathrm{~min}$ of recording, the resistance was significantly $(p=0.0029)$ reduced to $63 \pm 9.21 \%$ of the resistance measured at $0 \mathrm{~min}$. At the 32-min time point, there was a significant $(p=0.0002)$ difference in resistance between the neurons that received $\alpha$-syn monomers or vehicle to those where $\alpha$-syn aggregates were introduced. $\boldsymbol{E}$, Mean resting membrane potential plotted against time for control (vehicle) neurons and neurons that had either $\alpha$-syn aggregates or monomers introduced. Under all experimental conditions, the neurons slowly depolarized over the time course of the recording. For vehicle, the mean depolarization $(\Delta \mathrm{Vm})$ was $6.2 \pm 1.82 \mathrm{mV}$ after $32 \mathrm{~min}$ of recording $(p=0.0234)$, for $\alpha$-syn monomers $\Delta \mathrm{Vm}$ was $3.8 \pm 1.2 \mathrm{mV}(p=0.0938)$ and for $\alpha$-syn aggregates $\Delta \mathrm{Vm}$ was $6.8 \pm 1.85 \mathrm{mV}(p=0.0107)$. $\boldsymbol{F}$, Normalized firing rate plotted against time for control (vehicle) neurons and neurons that had either $\alpha$-syn aggregates or monomers introduced (firing rate was measured from naturalistic current injection). Data are normalized to the firing rate at time 0 (whole-cell break through). Despite depolarizing by a comparable amount to the control (vehicle) and monomer introduced neurons, $\alpha$-syn aggregates induced a significant reduction in the firing rate over time $(p=0.0020)$ consistent with the fall in input resistance. When comparing control (vehicle and $\alpha$-syn monomers) and $\alpha$-syn aggregates there was a significant difference in the firing rate at $32 \mathrm{~min}(p=0.0061)$. G, The same section of voltage trace taken during the injection of naturalistic current in example neurons injected with vehicle, $\alpha$-syn monomers, and $\alpha$-syn aggregates to illustrate the changes in firing pattern over the duration of recordings.

glibenclamide. There was no significant reduction in resistance or firing rate over the duration of recording when slices were incubated with glibenclamide suggesting that the action of $\alpha$-syn aggregates could least in part be mediated by KATP channels. The resistance at $32 \mathrm{~min}$ was $90 \pm 8.62 \%$ of the resistance at time 0 min (compare $63 \%$ without glibenclamide), which was not statistically significant $(p=0.25, n=6)$ from the resistance at time 0 . The firing rate was $80.14 \pm 12.89 \%$ of the rate at time 0 min (vs $42 \%$ without glibenclamide) which was not statistically significant from the firing rate at time $0 \min (p=0.353, n=6)$. Both sets of recorded neurons (vehicle and $\alpha$-syn aggregates) weakly depolarized over the 32 min of recording to a similar
A

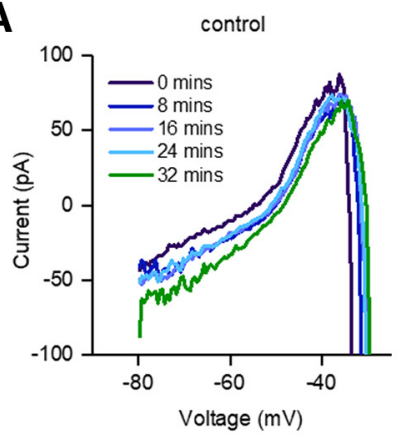

E

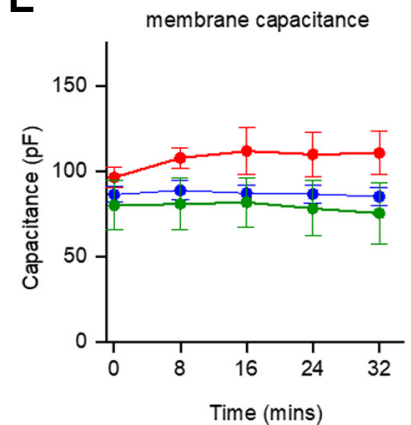

B

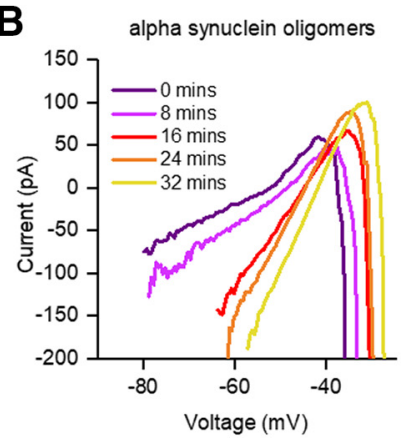

$\mathbf{F}$

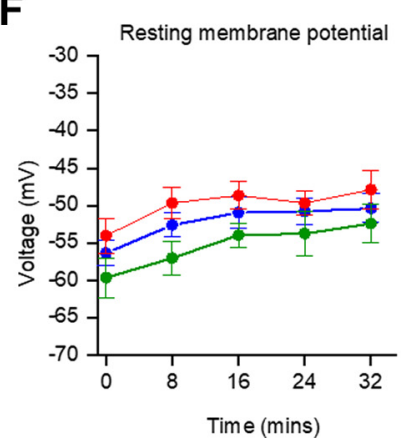

C

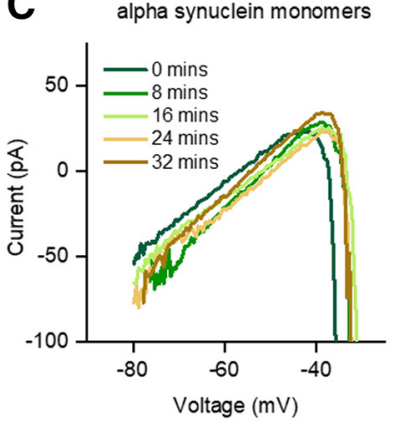

G

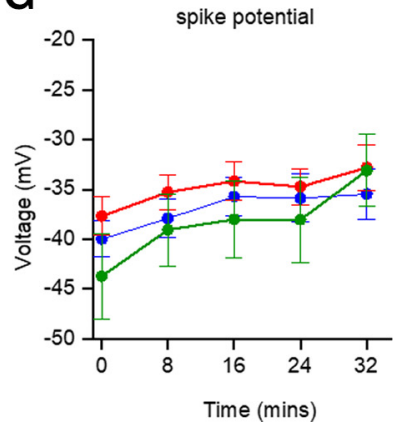

D membrane conductance

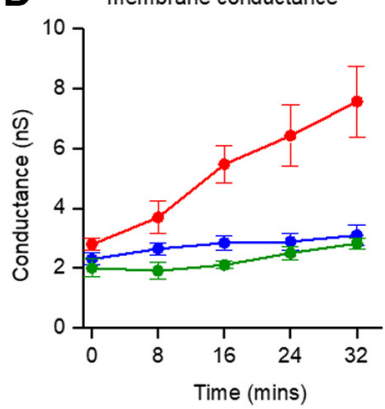

H

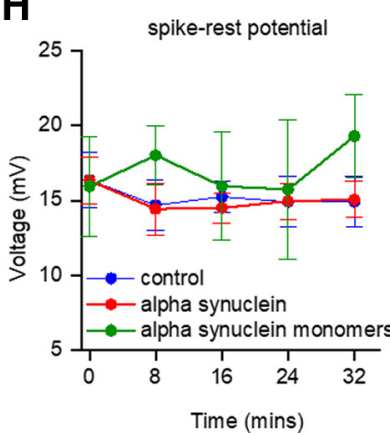

Figure 5. Intracellular diffusion of $\alpha$-syn aggregates results in a progressive conductance increase in the DIV curve. Example DIV curves from neurons that had control (vehicle, $\boldsymbol{A}), \alpha$-syn aggregates $(\boldsymbol{B})$, or monomeric $\alpha$-syn $(\boldsymbol{C})$ introduced. Each graph shows the DIV curve at 8-min time points throughout recordings (32 min). There is a marked increase in the gradient of the DIV (conductance) when oligomeric $\alpha$-syn was introduced. $\boldsymbol{D}-\boldsymbol{H}$, Extracted neuronal parameters averaged across recordings for the time periods during recordings ( $n=10$ for control, $n=11 \alpha$-syn aggregates and $n=6 \alpha$-syn, monomers). Capacitance (D) showed no significant change over time for all the experimental conditions. Although both control (vehicle) and monomeric $\alpha$-syn neurons showed a small increase in conductance $(E)$ over time ( $\sim 35 \%$ and $\sim 40 \%$ after $32 \mathrm{~min}$, respectively), the average conductance for the cells receiving $\alpha$-syn aggregates was almost threefold its initial value by $32 \mathrm{~min}(\sim 170 \%$ increase; $p=0.009)$. At 32 min, there was no significant $(p>0.9999)$ difference between the conductance of vehicle and $\alpha$-syn monomer treated neurons. In contrast, the conductance of $\alpha$-syn aggregate treated neurons was significantly $(p=0.0003)$ larger than vehicle treated neurons. Although both the resting potential $(\boldsymbol{F})$ and spike threshold $(\boldsymbol{G})$ slightly increased with time, the potential difference from resting potential to spike threshold remained constant with time. The decrease in excitability of the cells receiving $\alpha$-syn aggregates was therefore mediated by a conductance shunt rather than an increase in the relative threshold for spike initiation. 


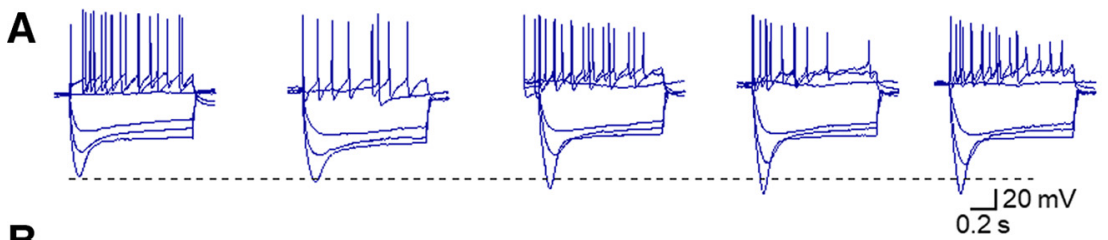

B
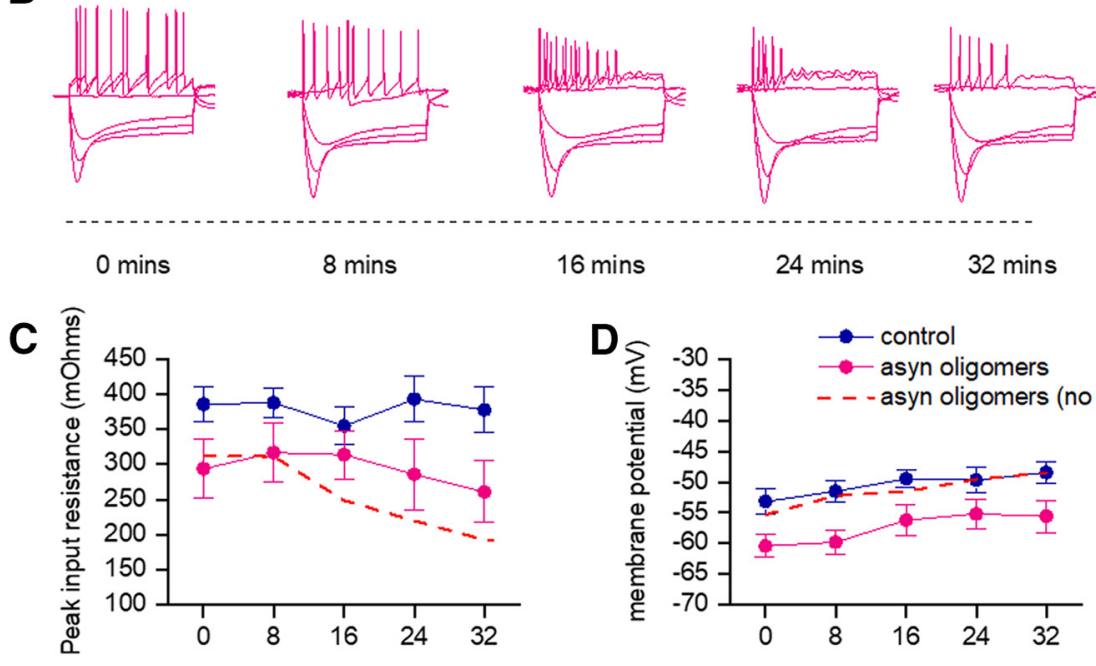

16 mins

24 mins

32 mins

E

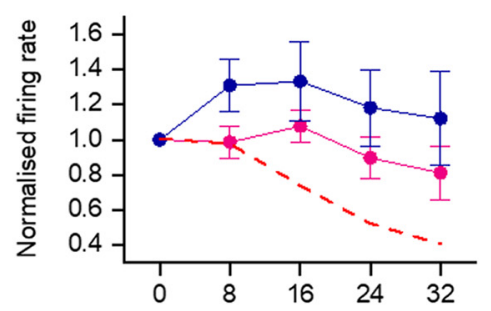

D $\rightarrow$ control

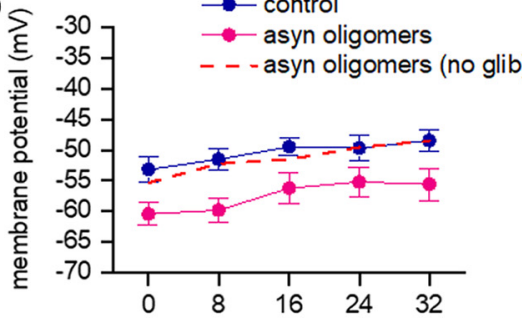

F
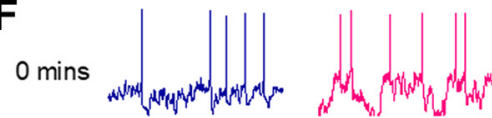

32 mins

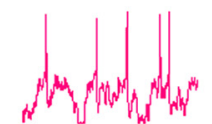

$0.5 \mathrm{~s}$

Figure 6. The effects of $\alpha$-syn aggregates on electrophysiological properties are reversed by glibenclamide. $\boldsymbol{A}$, SIV for an example control neuron (injected with vehicle) in the presence of the KATP channel inhibitor glibenclamide (1 $\mu \mathrm{M})$. Current steps (starting at $-300 \mathrm{pA}$ and rising by $100 \mathrm{pA}$ ) were injected until a regular firing pattern was induced. SIV traces are displayed at time points between whole-cell breakthrough $(0 \mathrm{~min})$ and the end of recording $(32 \mathrm{~min})$. The neuron remained stable for the duration of the recording with little change in the SIV. $\boldsymbol{B}$, As in $\boldsymbol{A}$, but with $\alpha$-syn aggregates (500 nM, final concentration) added to the internal recording solution. The decrease in input resistance and firing rate that was observed with $\alpha$-syn aggregates in control conditions was markedly reduced by the presence of glibenclamide. $\boldsymbol{C}$, Mean resistance measurements over time for control (vehicle) versus $\alpha$-syn aggregates in the presence of glibenclamide. The fall in resistance was reduced compared with that for $\alpha$-syn aggregate-injected neurons recorded in normal aCSF. $\boldsymbol{D}$, Mean resting membrane potential measurements plotted against time for vehicle versus $\alpha$-syn aggregates in the presence of glibenclamide. $\boldsymbol{E}$, Mean firing rate (measured from naturalistic current injection) plotted against time for vehicle versus $\alpha$-syn aggregates in the presence of glibenclamide. The fall in firing rate was reduced compared with that for $\alpha$-syn aggregate-injected neurons in normal aCSF. The red dotted line represents the effects observed with the introduction of $\alpha$-syn aggregates in the absence of glibenclamide, these data are repeated from Figure 4 and are intended to provide a visual reference to demonstrate the partial recovery.

degree to that of non-glibenclamide-treated slices (vehicle, $\Delta \mathrm{Vm} 4.66 \pm 2.6 \mathrm{mV}$ and $\alpha$-syn aggregates, $\Delta \mathrm{Vm}$ $5.5 \pm 2.05 \mathrm{mV}$; Fig. $6 E$ ).

We further investigated the effects of glibenclamide on the effects of $\alpha$-syn aggregates, using the DIV. The DIV curves in glibenclamide (vehicle) and in glibenclamide ( $\alpha$-syn aggregates) did not markedly change throughout the duration of the recordings (Fig. $7 A, B$ ). As observed previously (without glibenclamide) there was also no change in capacitance over the duration of the recordings (vehicle $p=0.0625, \alpha$-syn aggregates $p=0.0625$; Fig. 7C). In the presence of glibenclamide, there was no significant increase $(p=0.0625)$ in whole-cell conductance for vehicle receiving neurons over the duration of the recordings or only a small increase for $\alpha$-syn aggregate receiving neurons $(p=0.0313)$. After $32 \mathrm{~min}$, there was no significant $(p=0.3095)$ difference between the whole-cell conductance of neurons receiving vehicle and those receiving $\alpha$-syn aggregates (Fig. 7D,H). For all the other extracted parameters there was no difference between vehicle and $\alpha$-syn aggregate receiving neurons for the duration of recordings (Fig. 7E-G). Thus, as observed with SIV analysis, the KATP inhibitor glibenclamide significantly reduced the effects of $\alpha$-syn aggregates. 
A

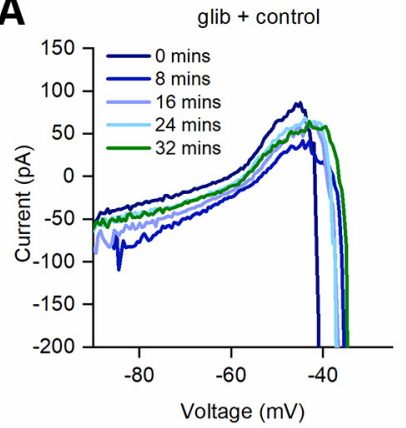

E

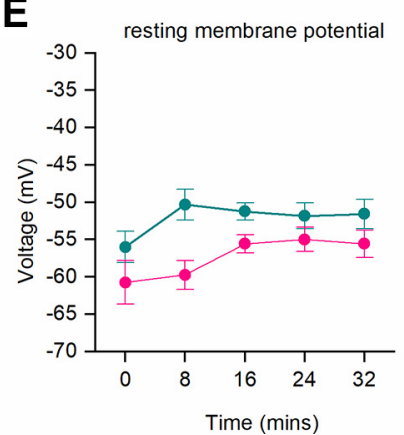

B

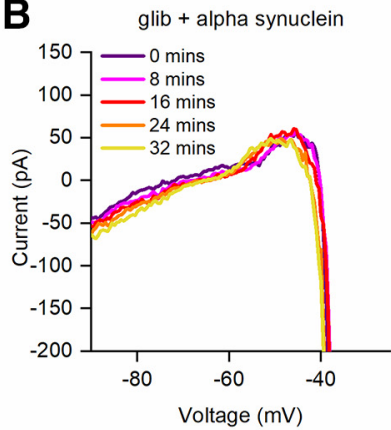

F

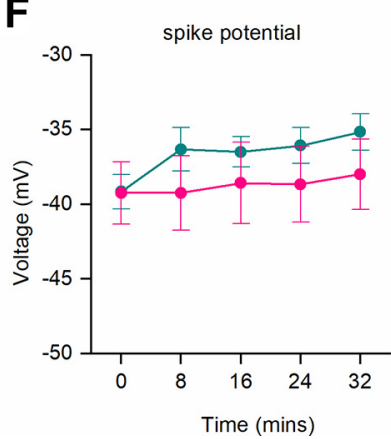

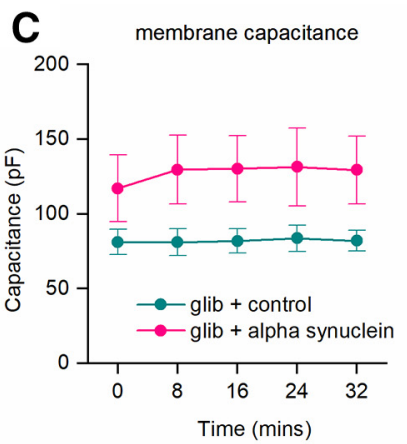

D membrane conductance
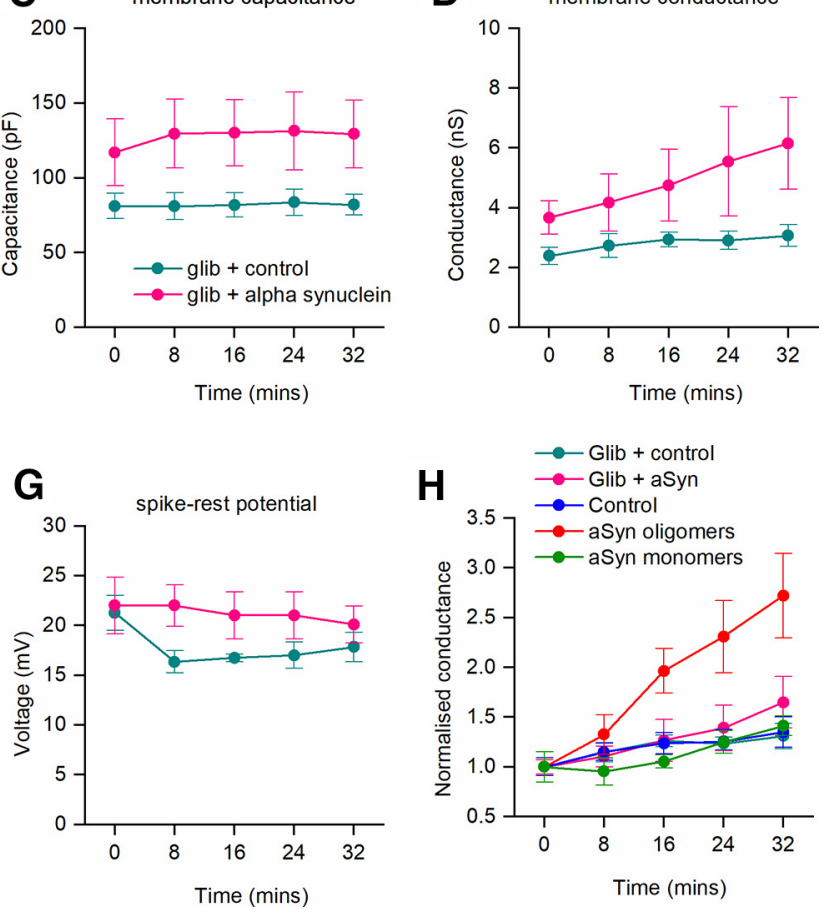

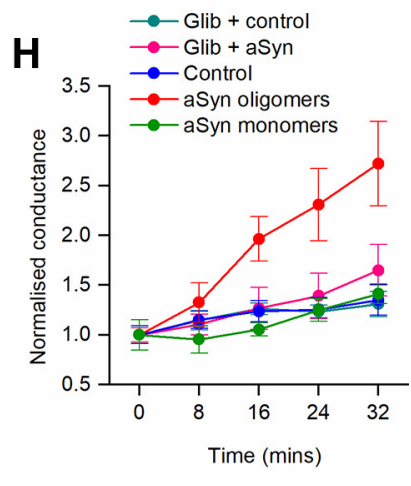

Figure 7. The KATP channel inhibitor glibenclamide blunts the effects of $\alpha$-syn aggregates on the DIV. Slices were incubated in glibenclamide $(1 \mu \mathrm{M})$ to block KATP channels. $\boldsymbol{A}$, Example DIV curves for a neuron that had vehicle introduced. $\boldsymbol{B}$, Example DIV curves for a neuron that had $\alpha$-syn aggregates introduced. For $\boldsymbol{A}, \boldsymbol{B}$, each graph shows the DIV curves at 8-min time points throughout recordings $(32 \mathrm{~min})$. There are no marked changes in the DIV across time for the two experimental conditions. $\boldsymbol{C}-\boldsymbol{H}$, Extracted neuronal parameters averaged across recordings for the time periods during recordings ( $n=6$ for vehicle, $n=6 \quad \alpha$-syn aggregates). Capacitance $(\boldsymbol{C})$ showed no significant change over time for all the experimental conditions. $\boldsymbol{D}$, There was a small increase in wholecell conductance for neurons with $\alpha$-syn aggregates, but the percentage change was closer to vehicle than to $\alpha$-syn aggregates without glibenclamide. Resting potential $(\boldsymbol{F})$ and spike threshold $(\boldsymbol{G})$ also slightly increased with time, the potential difference from rest potential to spike threshold remained constant with time. $\boldsymbol{H}$, Graph plotting the normalized conductance (normalized to the conductance at time $0 \mathrm{~min}$ ) for all experimental conditions. Using a Kruskal-Wallis ANOVA, we confirmed that both time and experimental condition had a significant effect on conductance $(p<0.0001$ and $p<0.0001$, respectively). At 32 min, the neurons with $\alpha$-syn aggregates injected have a significantly greater conductance than any of the other experimental conditions (vs control $p<0.0001$, vs monomer $p=0.0003$, vs glib + control $p<0.0001$, vs glib $+\alpha$-syn $p=0.0045$; Dunn's post hoc analysis). No other experimental conditions were significantly different from each other.

\section{Discussion}

In this study, we have used whole-cell patch-clamp recording to introduce a known concentration and form of aggregated $\alpha$-syn into single SNpc DNs in acutely isolated mouse slices. Although it is possible, using emerging technologies, to isolate only the oligomeric fractions (Kumar et al., 2020), we have not utilized such methods. Thus, we cannot exclude the possibility that some small fibrils are present in our $\alpha$-syn oligomer samples. While the majority of the species in our $\alpha$-syn samples appeared to have an oligomeric structure (Fig. 3) other forms of small aggregates maybe present (for example, small $\sim 50-$ $\mathrm{nm}$ fibrils; Polinski et al. 2018). We have therefore used the term $\alpha$-syn aggregates rather than $\alpha$-syn oligomers. As outlined in previous studies (Kaufmann et al., 2016; Hill et al., 2019) delivery of species via the patch pipette has a number of advantages, the main one being that it is possible to measure early changes in the properties of a single neuron in a network that is free from pathology. This method also bypasses any slow uptake steps and each neuron acts as its own control, as at early time points only low concentrations of the species will have diffused into the neuron. Here, we have introduced 500 nм $\alpha$-syn aggregates, which is a concentration in line with previous studies (see Kaufmann et al., 2016). However, as noted in the methods, the actual concentration of aggregates will be considerably lower. It would be interesting in future experiments to investigate whether there is a concentration dependent effect of $\alpha$-syn aggregates, with, for example, lower concentrations having a slower onset (see Hill et al., 2019).

We used both SIV (with step current injections) and the DIVs (with naturalistic current injection) to extract neuronal parameters. Both approaches measured a marked increase in whole-cell conductance (fall in neuronal resistance), which occurred between 8 and $16 \mathrm{~min}$ after whole-cell break through. There were no significant changes in membrane potential, cell capacitance or spike threshold between neurons receiving $\alpha$-syn aggregates, $\alpha$-syn monomers, or vehicle. The increase in conductance significantly reduced the firing rate, both induced (by current injection) and spontaneous, and abolished rebound firing following hyperpolarizing current steps. The 
increase in conductance increased the linearity of the DIV curve effectively shunting the depolarization-activated conductance increase just below threshold that was present in some neurons at $0 \mathrm{~min}$ (whole-cell breakthrough).

Obtaining similar results using two independent methods for the extraction of neuronal parameters strengthens the robustness of the observation that $\alpha$-syn aggregates increase whole-cell conductance. There were minor differences in the precise values of the neuronal parameters extracted using DIV compared with those extracted with SIV, which is to be expected given the different conductance states of the cell in the two protocols. For the DIV, the voltage varies across a wide range during naturalistic stimulation (mimicking in vivo synaptic activity) activating and inactivating a variety of conductances. In contrast, the SIV extracts parameters over a much smaller range of voltages from essentially quiescent cells. For example, if the DIV and SIV curves are compared, the DIV conductances are initially lower (at $0 \mathrm{~min}$ ) for all of the experimental conditions (vehicle, aggregates and monomers) and then increase. This could be interpreted as one or more of the conductances being slightly more inactivated during the DIV protocol. It could then be that this inactivation is relieved as the neuronal resting potential slowly depolarizes during the recording. Alternatively, it could be that another conductance activates as the neuron depolarizes, but in a range that is not accessed by the hyperpolarizing SIV current steps. In either case, a different range of voltages is probed between DIV and SIV and so differences in the extracted parameters will occur. The fact that the same significant conductance increase (resistance decrease) is clearly seen in these two very different protocols attests to the robustness of the effect of $\alpha$-syn on these neurons.

\section{The increase in whole-cell conductance is reduced by the KATP channel blocker glibenclamide}

The effects of the KATP inhibitor glibenclamide (Light and French, 1994) suggest the involvement of KATP channels (but see caveats below). KATP channels are inwardly rectifying $\mathrm{K}^{+}$-selective ion channels that are inhibited by intracellular ATP. KATP channels provide a link between the energy state of cells and their electrical activity acting as a metabolically controlled "brake on excitation." A decrease in sub-membrane ATP levels and an accompanying rise in ADP concentration (during activity) triggers KATP channel opening dropping neuronal resistance and hyperpolarizing the membrane potential (Stanford and Lacey, 1995; Seino, 1999; Haller et al., 2001). It is well established that SNpc DNs express KATP channels (Liss et al., 2005; Schiemann et al., 2012). The lack of effect of the KATP inhibitor glibenclamide under control conditions suggests that few KATP channels are open at rest. This is perhaps not surprising as there was $2 \mathrm{~mm}$ ATP in the intracellular patch solution, although some KATP channels have a low affinity for ATP and would still be open with this level of ATP (see Allen and Brown, 2004). The effects of $\alpha$-syn aggregates on conductance and its inhibition by glibenclamide are consistent with the opening of KATP channels (but see below). These effects occurred in the presence of $2 \mathrm{~mm}$ intracellular ATP suggesting a direct effect of $\alpha$-syn aggregates on the KATP channel, rather than via a reduction in intracellular ATP concentration (by, for example, mitochondrial dysfunction). KATP channel openers like diazoxide have previously been shown to act by open KATP channels in the presence of ATP (Schwanstecher et al., 1998) so it is possible that $\alpha$-syn aggregates could have a similar action.

The effects on cell conductance and its prevention with glibenclamide are consistent with the $\alpha$-syn aggregates increasing KATP channel open probability. However, there was little or no associated membrane potential hyperpolarization (measured with SIV and with DIV), which has been observed in most reports of KATP channel activation (see Stanford and Lacey, 1995; Allen and Brown, 2004). The equilibrium potential for $\mathrm{K}^{+}$is $\sim-95 \mathrm{mV}$, so opening of KATP channels would be expected to produce a large hyperpolarization of the membrane potential (as the resting potential at time 0 was $\sim-55 \mathrm{mV}$ ). However, the membrane potential hyperpolarization could possibly be counteracted by the opposing activation of hyperpolarization-activated, cyclic nucleotide-gated HCN channels I(h) which have a reversal potential between -40 to $-30 \mathrm{mV}$ (Mayer and Westbrook, 1983). It may be that the opening of a relatively small number of KATP channels changes the input resistance/conductance of neurons but is insufficient to change the membrane potential against the responsive reaction of $\mathrm{I}(\mathrm{h})$. In contrast opening of many KATP channels will overcome the effects of $I(h)$ leading to robust membrane hyperpolarization, which as reported for SNpc DNs by lowering intracellular ATP concentration or using KATP channel opening drugs (Stanford and Lacey, 1995).

The inhibitory effects of glibenclamide on $\alpha$-syn responses are consistent with the opening of KATP channels but additional evidence is required to provide definitive evidence for a role for KATP channels in the effects of $\alpha$-syn aggregates. One possible approach is to occlude the effects of $\alpha$-syn aggregates using a pharmacological opener of KATP channels (such as diazoxide). However, these experiments maybe be difficult to interpret. The KATP channel opener will markedly reduce resistance and hyperpolarize the recorded neuron. This may occlude the effects of $\alpha$-syn aggregates simply by shunting the neuron and moving it closer to $E_{K}$ rather than acting through the same channel. Other possible approaches include the use of transgenic animals, where the KATP gene has been deleted, or using cell lines expressing specific KATP channels. Alternate possibilities for the lack of membrane hyperpolarization include the opening of other channels that depolarize the neuron. For example, it has been reported that $\alpha$-syn itself can form non-selective cation channels (Mironov, 2015).

It took on average between 8 and 16 min of dialysis for the effects of $\alpha$-syn aggregates on whole-cell conductance to become significant. It may be that the concentration within the neuron has to reach a sufficient level to open membrane channels. For comparison introduction of aggregated tau (444 nм) into pyramidal neurons started to have effects on the action potential within the first 10 min (Hill et al., 2019). In this study, we chose to focus on the effects of introducing $\alpha$-syn aggregates into 
substantia nigra DNs. Given that the neighboring population of VTA DNs have differences in their KATP channel expression (Liss et al., 2005), it might be expected that $\alpha$-syn aggregates may have a different effect in these cells (which would additional evidence for a role of KATP channels). This may contribute to the varying vulnerability of these two dopaminergic nuclei in diseases like PD.

The accumulation of $\alpha$-syn aggregates in SN DNs during PD progression could potentially result in the prolonged activation of membrane channels (such as KATP) which will chronically reduce electrical activity, the amount of dopamine released (Patel et al., 2011) and will be detrimental to neuron function. If KATP channels are involved then there will be a loss of the metabolic feedback mechanism. Consistent with this KATP involvement, there is retrospective epidemiological evidence of a reduced risk for $P D$ in type 2 diabetic patients that were treated with KATP inhibitors (Schernhammer et al., 2011; Wahlqvist et al., 2012; Cereda et al., 2013; Lu et al., 2014; Brauer et al., 2015).

In this study, we have combined electrophysiological recording with detailed and thorough analysis to characterize the effects of introducing aggregated $\alpha$-syn directly into single mouse DNs in the substantia nigra. Aggregated $\alpha$-syn caused a significant increase in conductance and decrease in firing rate without altering the resting membrane potential, capacitance or spike threshold. Changes to conductance and firing rate occurred 8-16 min after whole-cell breakthrough and were specific to aggregates (they were not observed when monomeric $\alpha$-syn was introduced). The effects could be prevented by preincubating the slices in KATP inhibitor glibenclamide, despite the high concentration of ATP present in the patch electrode. This suggests that aggregated $\alpha$-syn may increase the opening probability of KATP channels, resulting in an increase in conductance, a reduction in neuronal excitability and likely also a decrease in dopamine release and overall cell function.

\section{References}

Alderson TR, Markley JL (2013) Biophysical characterization of $\alpha$-synuclein and its controversial structure. Intrinsically Disord Proteins 1:18-39.

Allen TG, Brown DA (2004) Modulation of the excitability of cholinergic basal forebrain neurones by KATP channels. J Physiol 554:353-370.

Badel L, Lefort S, Brette R, Petersen C, Gerstner W, Richardson M (2008a) Dynamic I-V curves are reliable predictors of naturalistic pyramidal-neuron voltage traces. J Neurophysiol 99:656-666.

Badel L, Lefort S, Berger T, Petersen C, Gerstner W, Richardson M (2008b) Extracting non-linear integrate-and-fire models from experimental data using dynamic I-V curves. Biol Cybern 99:361370.

Bernheimer H, Birkmayer W, Hornykiewicz O, Jellinger K, Seitelberger F (1973) Brain dopamine and the syndromes of Parkinson and Huntington. Clinical, morphological and neurochemical correlations. J Neurol Sci 20:415-455.

Bengoa-Vergniory N, Roberts R, Wade-Martins R, AlegreAbarrategui J (2017) Alpha-synuclein oligomers: a new hope. Acta Neuropathol 134:819-838.

Bezanson J, Edelman A, Karpinski S, Shah VB (2017) Julia: a fresh approach to numerical computing. SIAM Rev 59:65-98.

Brauer R, Bhaskaran K, Chaturvedi N, Dexter DT, Smeeth L, Douglas I (2015) Glitazone treatment and incidence of Parkinson's disease among people with diabetes: a retrospective cohort study. PLoS Med 12:e1001854

Burré J, Sharma M, Tsetsenis T, Buchman V, Etherton MR, Südhof TC (2010) alpha synuclein promotes SNARE-complex assembly in vivo and in vitro. Science 329:1663-1667.

Cereda E, Barichella M, Pedrolli C, Klersy C, Cassani E, Caccialanza R, Pezzoli G (2013) Diabetes and risk of Parkinson's disease. Mov Disord 28:257.

Damier P, Hirsch EC, Agid Y, Graybiel AM (1999) The substantia nigra of the human brain. II. Patterns of loss of dopamine-containing neurons in Parkinson's disease. Brain 122:1437-1448.

Grace AA, Onn SP (1989) Morphology and electrophysiological properties of immunocytochemically identified rat dopamine neurons recorded in vitro. J Neurosci 9:3463-3481.

Haller M, Mironov SL, Karschin A, Richter DW (2001) Dynamic activation of K(ATP) channels in rhythmically active neurons. J Physiol 537:69-75.

Harris NC, Constanti A (1995) Mechanism of block by ZD 7288 of the hyperpolarization-activated inward rectifying current in guinea pig substantia nigra neurons in vitro. J Neurophysiol 74:2366-2378.

Harrison PM, Badel L, Wall MJ, Richardson MJE (2015) Experimentally verified parameter sets for modelling heterogeneous neocortical pyramidal-cell populations. PLoS Comput Biol 11:e1004165.

Hill E, Karikari TK, Moffat G, Richardson MJE, Wall MJ (2019) Introduction of tau oligomers into cortical neurons alters action potential dynamics and disrupts synaptic transmission and plasticity. eNeuro 6:ENEURO.0166-19.2019.

Iwai A, Masliah E, Yoshimoto M, Ge N, Flanagan L, de Silva HA, Kittel A, Saitoh T (1995) The precursor protein of non-A beta component of Alzheimer's disease amyloid is a presynaptic protein of the central nervous system. Neuron 14:467-475.

Jakes R, Spillantini MG, Goedert M (1994) Identification of two distinct synucleins from human brain. FEBS Lett 345:27-32.

Kaufmann TJ, Harrison P, Richardson MJE, Pinheiro TJT, Wall MJ (2016) Intracellular injection of soluble alpha synuclein oligomers reduces pyramidal cell input resistance and firing rate. J Physiol 594:2751-2772.

Kumar ST, Donzelli S, Chiki A, Syed MMK, Lashuel HA (2020) A simple, versatile and robust centrifugation-based filtration protocol for the isolation and quantification of $\alpha$-synuclein monomers, oligomers and fibrils: towards improving experimental reproducibility in $\alpha$-synuclein research. J Neurochem 153:103-119.

Jiang C, Haddad GG (1997) Modulation of K+ channels by intracellular ATP in human neocortical neurons. J Neurophysiol 77:93-102.

Krashia P, Martini A, Nobili A, Aversa D, D'Amelio M, Berretta N, Guatteo E, Mercuri NB (2017) On the properties of identified dopaminergic neurons in the mouse substantia nigra and ventral tegmental area. Eur J Neurosci 45:92-105.

Lacey MG, Mercuri NB, North RA (1989) Two cell types in rat substantia nigra zona compacta distinguished by membrane properties and the actions of dopamine and opioids. J Neurosci 9:12331241.

Lashuel HA, Overk CR, Oueslati A, Masliah E (2013) The many faces of $\alpha$ synuclein: from structure and toxicity to therapeutic target. Nat Rev Neurosci 14:38-48.

Light PE, French RJ (1994) Glibenclamide selectively blocks ATPsensitive $\mathrm{K}+$ channels reconstituted from skeletal muscle. Eur J Pharmacol 259:219-222.

Liss B, Haeckel O, Wildmann J, Miki T, Seino S, Roeper J (2005) KATP channels promote the differential degeneration of dopaminergic midbrain neurons. Nat Neurosci 8:1742-1751.

Lu L, Fu DL, Li HQ, Liu AJ, Li JH, Zheng GQ (2014) Diabetes and risk of Parkinson's disease: an updated meta-analysis of case-control studies. PLoS One 9:e85781.

Mahul-Mellier AL, Burtscher J, Maharjan N, Weerens L, Croisier M, Kuttler F, Leleu M, Knott GW, Lashuel HA (2020) The process of Lewy body formation, rather than simply alpha-synuclein fibrillization, is the major driver of neurodegeneration in synucleinopathies. Proc Natl Acad Sci USA 117:4971-4982. 
Mayer ML, Westbrook GL (1983) A voltage-clamp analysis of inward (anomalous) rectification in mouse spinal sensory ganglion neurones. J Physiol 340:19-45.

Michel PP, Hirsch EC, Hunot S (2016) Understanding dopaminergic cell death pathways in Parkinson disease. Neuron 90:675-691.

Mironov S (2015) $\alpha$-Synuclein forms non-selective cation channels and stimulates ATP-sensitive potassium channels in hippocampal neurons. J Physiol 593:145-159.

Murphy DD, Rueter SM, Trojanowski JQ, Lee VMY (2000) Synucleins are developmentally expressed, and $\alpha$-synuclein regulates the size of the presynaptic vesicular pool in primary hippocampal neurons. J Neurosci 20:3214-3220.

Neuhoff H, Neu A, Liss B, Roeper J (2002) I(h) channels contribute to the different functional properties of identified dopaminergic subpopulations in the midbrain. J Neurosci 22:1290-1302.

Patel JC, Witkovsky P, Coetzee WA, Rice ME (2011) Subsecond regulation of striatal dopamine release by pre-synaptic KATP channels. J Neurochem 118:721-736.

Polinski NK, Volpicelli-Daley LA, Sortwell CE, Luk KC, Cremades N, Gottler LM, Froula J, Duffy MF, Lee VMY, Martinez TN, Dave KD (2018) Best practices for generating and using alpha-synuclein pre-formed fibrils to model Parkinson's disease in rodents. J Parkinsons Dis 8:303-322.

Richards CD, Shiroyama T, Kitai ST (1997) Electrophysiological and immunocytochemical characterization of GABA and dopamine neurons in the substantia nigra of the rat. Neuroscience 80:545-557.

Schernhammer E, Hansen J, Rugbjerg K, Wermuth L, Ritz B (2011) Diabetes and the risk of developing Parkinson's disease in Denmark. Diabetes Care 34:1102-1108.

Schiemann J, Schlaudraff F, Klose V, Bingmer M, Seino S, Magill P, Zaghloul KA, Schneider G, Liss B, Roeper J (2012) K-ATP channels in dopamine substantia nigra neurons control bursting and novelty-induced exploration. Nat Neurosci 15:1272-1280.
Schwanstecher M, Sieverding C, Dörschner H, Gross I, AguilarBryan L, Schwanstecher C, Bryan J (1998) Potassium channel openers require ATP to bind to and act through sulfonylurea receptors. EMBO J 17:5529-5535.

Seino S (1999) ATP-sensitive potassium channels: a model of heteromultimeric potassium channel/receptor assemblies. Annu Rev Physiol 61:337-362.

Spillantini MG, Schmidt ML, Lee VMY, Trojanowski JQ, Jakes R, Goedert M (1997) $\alpha$-Synuclein in Lewy bodies. Nature 388:839840.

Stanford IM, Lacey MG (1995) Regulation of a potassium conductance in rat midbrain dopamine neurons by intracellular adenosine triphosphate (ATP) and the sulfonylureas tolbutamide and glibenclamide. J Neurosci 15:4651-4657.

Thakur P, Luk K, Roeper J (2019) Selective K-ATP channel-dependent loss of pacemaking in vulnerable nigrostriatal dopamine neurons by $\alpha$-synuclein aggregates. bioRxiv 842344 doi: 10.1101/ 842344.

Wahlqvist M, Lee MS, Hsu CC, Chuang SY, Lee J, Tsai HN (2012) Metformin-inclusive sulfonylurea therapy reduces the risk of Parkinson's disease occurring with Type 2 diabetes in a Taiwanese population cohort. Parkinsonism Relat Disord 18:753758.

Winner B, Jappelli R, Maji SK, Desplats PA, Boyer L, Aigner S, Hetzer C, Loher T, Vilar M, Campioni S, Tzitzilonis C, Soragni A, Jessberger S, Mira H, Consiglio A, Pham E, Masliah E, Gage FH, Riek R (2011) In vivo demonstration that $\alpha$-synuclein oligomers are toxic. Proc Natl Acad Sci USA 108:4194-4199.

Zhang Q, Li C, Zhang T, Ge Y, Han X, Sun S, Ding J, Lu M, Hu G (2018) Deletion of Kir6.2/SUR1 potassium channels rescues diminishing of DA neurons via decreasing iron accumulation in PD. Mol Cell Neurosci 92:164-176. 